ESAIM: M2AN

Vol. 40, No 6, 2006, pp. 1101-1125

DOI: $10.1051 / \mathrm{m} 2 \mathrm{an}: 2007003$
ESAIM: Mathematical Modelling and Numerical Analysis

www.edpsciences.org/m2an

\title{
NUMERICAL SIMULATION OF A PULSATILE FLOW THROUGH A FLEXIBLE CHANNEL
}

\author{
Cornel Marius Murea ${ }^{1}$
}

\begin{abstract}
An algorithm for approximation of an unsteady fluid-structure interaction problem is proposed. The fluid is governed by the Navier-Stokes equations with boundary conditions on pressure, while for the structure a particular plate model is used. The algorithm is based on the modal decomposition and the Newmark Method for the structure and on the Arbitrary Lagrangian Eulerian coordinates and the Finite Element Method for the fluid. In this paper, the continuity of the stresses at the interface was treated by the Least Squares Method. At each time step we have to solve an optimization problem which permits us to use moderate time step. This is the main advantage of this approach. In order to solve the optimization problem, we have employed the Broyden, Fletcher, Goldforb, Shano Method where the gradient of the cost function was approached by the Finite Difference Method. Numerical results are presented.
\end{abstract}

Mathematics Subject Classification. 74F10, 75D05, 65M60.

Received: January 15, 2005. Revised: June 1 and November 15, 2006.

\section{INTRODUCTION}

We consider a pulsatile incompressible flow through a channel with elastic walls. Following [29], the therm pulsatility means the rapid increase and decrease of the flow rate in a first phase, followed by a longer phase where the flow rate is small. This kind of fluid-structure interaction arises in car industries, for example the dynamic behavior of a hydraulic shock absorber [22] or in the design of sensors subject to large acceleration during impact [20] or in bio-mechanics, for example, the interaction between a bio-fluid and a living tissue [28].

The mathematical model which governs the fluid is the unsteady Navier-Stokes equations with boundary condition on the pressure. For the structure, a particular plate model is used.

The most frequently, the fluid-structure interaction problems are solved numerically by partitioned procedures, i.e. the fluid and the structure equations are solved separately.

There are different strategies to discretize in time the unsteady fluid-structure interaction problem. A family of explicit algorithms known also as staggered was successfully employed for the aeroelastic applications [11]. As it shown in [22] and [26], the staggered algorithms are unstable when the structure is light and its density is comparable to that of its fluid, such in the bio-mechanics applications. For a simplified fluid-structure problem, the unconditionally instability of the explicit algorithms is proved in [3].

Keywords and phrases. Fluid-structure interaction, Navier-Stokes equations, Arbitrary Lagrangian Eulerian Method.

1 Laboratoire de Mathématiques, Informatique et Applications, Université de Haute-Alsace, 4, rue des Frères Lumière, 68093 Mulhouse Cedex, France; cornel.murea@uha.fr

(c) EDP Sciences, SMAI 2007 
In order to obtain unconditionally stable algorithms, at each time step we have to solve a non-linear fluidstructure coupled system. This can be done by using: fixed point strategies [13,22, 26, 28], Newton Method where the gradient is approached by Finite Differences [30], quasi-Newton Method [14], Newton Method with exact Jacobian [12]. The starting point for these iterative methods at the current time step is computed by extrapolating the solutions at the previous time steps.

We will see in the numerical tests presented in this paper that the solution at the previous time step, which is used as a starting point for the next time step, it is not close to the solution at the current time step. Such phenomena is amplified during the phase when the flow rate increase or decrease rapidly or if we increase the time step.

The fixed point and Newton like algorithms are not suitable in this case, since these methods diverge if the starting point is not sufficiently close to the solution.

In this paper, the continuity of the stresses at the interface will be treated by the Least Squares Method and at each time step we have to solve an optimization problem which is less sensitive to the choice of the starting point and it permits us to use moderate time step. This is the main advantage of this approach.

The outline of this paper is as follows. In Section 1 the approximation of the structure by the modal decomposition and Newmark scheme is presented. The Arbitrary Lagrangian Eulerian Method for NavierStokes equations in moving domain is detailed in Section 2. The coupled fluid-structure algorithm is introduced in Section 3. In order to solve the optimization problem at each time step, we have employed the Broyden, Fletcher, Goldforb, Shano Method and the gradient of the cost function was approached by the Finite Difference Method. Numerical results are presented and discussed in Section 4. The last section is devoted to some concluding remarks.

\section{Approximation of the structure}

\subsection{Strong equations of the structure}

The following system was obtained from the equations of a linear elastic, homogeneous, isotropic plate (see [10]) which is parallel to the plane $O x_{1} x_{3}$, under the hypothesis that the vertical displacement is independent of $x_{3}$.

Let $L>0$ denote the length, $h^{S}>0$ the thickness, $\rho^{S}>0$ the mass density, $E>0$ the Young modulus, $0<\nu \leq 0.5$ the Poisson ratio, $T>0$ the length of the time interval, $\eta:(0, L) \times(0, T) \rightarrow \mathbb{R}$ the applied transverse force per unit area, $u^{0}:(0, L) \rightarrow \mathbb{R}$ the initial displacement, $\dot{u}^{0}:(0, L) \rightarrow \mathbb{R}$ the initial velocity.

The problem is to find the transverse displacement $u:[0, L] \times[0, T] \rightarrow \mathbb{R}$ such that

$$
\begin{aligned}
& \rho^{S} h^{S} \frac{\partial^{2} u}{\partial t^{2}}\left(x_{1}, t\right)+\frac{E\left(h^{S}\right)^{3}}{12\left(1-\nu^{2}\right)} \frac{\partial^{4} u}{\partial x_{1}^{4}}\left(x_{1}, t\right)=\eta\left(x_{1}, t\right), \quad\left(x_{1}, t\right) \in(0, L) \times(0, T) \\
& u(0, t)=0, \frac{\partial u}{\partial x_{1}}(0, t)=0, \quad t \in(0, T) \\
& u(L, t)=0, \frac{\partial u}{\partial x_{1}}(L, t)=0, \quad t \in(0, T) \\
& u\left(x_{1}, 0\right)=u^{0}\left(x_{1}\right), \quad x_{1} \in(0, L) \\
& \frac{\partial u}{\partial t}\left(x_{1}, 0\right)=\dot{u}^{0}\left(x_{1}\right), \quad x_{1} \in(0, L)
\end{aligned}
$$

The above model is suitable for the flat thin structures. The time derivative of the angular momentum $\frac{\rho^{S}\left(h^{S}\right)^{3}}{12} \frac{\partial^{4} u}{\partial t^{2} \partial x_{1}^{2}}$ was neglected since the structure is thin and consequently $\left(h^{S}\right)^{3}$ is very small. On the contrary, the factor $\frac{E\left(h^{S}\right)^{3}}{12\left(1-\nu^{2}\right)}$ could not be neglected because the Young modulus $E$ is in general a big number. 
In $[28,29]$, for numerical simulations of the blood flow in arteries, the vascular wall was modeled as a axisymmetric membrane

$$
\rho^{S} h^{S} \frac{\partial^{2} u}{\partial t^{2}}\left(x_{1}, t\right)-h^{S} G k \frac{\partial^{2} u}{\partial x_{1}^{2}}\left(x_{1}, t\right)+\frac{E h^{S}}{12\left(1-\nu^{2}\right) R^{2}} u\left(x_{1}, t\right)=\eta\left(x_{1}, t\right) .
$$

Some authors add the visco-elastic term $\frac{\partial^{3} u}{\partial x_{1}^{2} \partial t}$ to the membrane model in order to obtain a priori estimation of an energy [23], or to regularize the solution [4], or to stabilize the numerical schemes [7].

\subsection{Natural frequencies and normal mode shapes}

This subsection follows the general reference [5], Volume 7, Chapter XV and the particular Example 5.3, p. 192 from the same reference. Another general reference in this topic is [21].

We denote by $0<s_{0}<\ldots<s_{i}<\ldots$ the solutions of the equation

$$
\cos (s) \cosh (s)=1, \quad s>0
$$

We set $a_{i}=s_{i} / L$. For each $i \in \mathbb{N}$ there exists a unique normal mode shape $\phi_{i} \in \mathcal{C}^{4}([0, L])$ such that

$$
\begin{aligned}
& \phi_{i}^{\prime \prime \prime \prime}\left(x_{1}\right)=\left(a_{i}\right)^{4} \phi_{i}\left(x_{1}\right), \quad x_{1} \in(0, L) \\
& \phi_{i}(0)=\frac{\partial \phi_{i}}{\partial x_{1}}(0)=0, \\
& \phi_{i}(L)=\frac{\partial \phi_{i}}{\partial x_{1}}(L)=0, \\
& \int_{0}^{L} \phi_{i}^{2}\left(x_{1}\right) \mathrm{d} x_{1}=1 .
\end{aligned}
$$

Let $\omega_{i}=\left(\frac{s_{i}}{L}\right)^{2} \sqrt{\frac{E\left(h^{S}\right)^{3}}{12\left(1-\nu^{2}\right) \rho^{S} h^{S}}}$ be the $i$ th natural frequency associated with $\phi_{i}$.

The normal mode shapes $\phi_{i}$ for $i \in \mathbb{N}$ form an orthonormal basis of $L^{2}(0, L)$. There exists a unique decomposition of $\eta$ of the form

$$
\eta\left(x_{1}, t\right)=\sum_{i \geq 0} \alpha_{i}(t) \phi_{i}\left(x_{1}\right)
$$

The problem (1.1)-(1.5) has a solution of the form

$$
u\left(x_{1}, t\right)=\sum_{i \geq 0} q_{i}(t) \phi_{i}\left(x_{1}\right)
$$

where $q_{i}$ is the solution of the second order differential equation

$$
\begin{aligned}
& q_{i}^{\prime \prime}(t)+\omega_{i}^{2} q_{i}(t)=\frac{1}{\rho^{S} h^{S}} \alpha_{i}(t), \quad t \in(0, T) \\
& q_{i}(0)=\int_{0}^{L} u^{0}\left(x_{1}\right) \phi_{i}\left(x_{1}\right) \mathrm{d} x_{1} \\
& q_{i}^{\prime}(0)=\int_{0}^{L} \dot{u}^{0}\left(x_{1}\right) \phi_{i}\left(x_{1}\right) \mathrm{d} x_{1} .
\end{aligned}
$$




\subsection{The Newmark Method}

We recall the Newmark Method employed to approximate second order systems of ordinary differential equations.

Let $N \in \mathbb{N}^{*}$ be the number of time steps and $\Delta t=T / N$ the time step. We set $t_{n}=n \Delta t$ for $n=0,1, \ldots, N$. We denote $\alpha_{i}^{n}=\alpha_{i}\left(t_{n}\right)$ and let $q_{i}^{n}, \dot{q}_{i}^{n}, \ddot{q}_{i}^{n}$ be approximations of $q_{i}\left(t_{n}\right), q_{i}^{\prime}\left(t_{n}\right), q_{i}^{\prime \prime}\left(t_{n}\right)$ respectively.

Knowing $q_{i}^{n}, \dot{q}_{i}^{n}, \ddot{q}_{i}^{n}$ and $\alpha_{i}^{n+1}$, find $q_{i}^{n+1}, \dot{q}_{i}^{n+1}, \ddot{q}_{i}^{n+1}$ such that:

$$
\begin{aligned}
& \ddot{q}_{i}^{n+1}+\omega_{i}^{2} q_{i}^{n+1}=\frac{1}{\rho^{S} h^{S}} \alpha_{i}^{n+1} \\
& \dot{q}_{i}^{n+1}=\dot{q}_{i}^{n}+\Delta t\left[(1-\delta) \ddot{q}_{i}^{n}+\delta \ddot{q}_{i}^{n+1}\right] \\
& q_{i}^{n+1}=q_{i}^{n}+\Delta t \dot{q}_{i}^{n}+(\Delta t)^{2}\left[\left(\frac{1}{2}-\theta\right) \ddot{q}_{i}^{n}+\theta \ddot{q}_{i}^{n+1}\right]
\end{aligned}
$$

where $\delta$ and $\theta$ are two real parameters.

Substituting (1.15) into (1.13) results in an equation that may be solved for $\ddot{q}_{i}^{n+1}$ :

$$
\left(1+\omega_{i}^{2}(\Delta t)^{2} \theta\right) \ddot{q}_{i}^{n+1}=\frac{1}{\rho^{S} h^{S}} \alpha_{i}^{n+1}-\omega_{i}^{2}\left[q_{i}^{n}+\Delta t \dot{q}_{i}^{n}+(\Delta t)^{2}\left(\frac{1}{2}-\theta\right) \ddot{q}_{i}^{n}\right]
$$

Once $\ddot{q}_{i}^{n+1}$ is determined, (1.14) and (1.15) serve to define $\dot{q}_{i}^{n+1}$ and $q_{i}^{n+1}$, respectively.

Following [5], Volume 9, p. 922, this method is unconditional stable for $2 \theta \geq \delta \geq 1 / 2$. It is first order accuracy if $\delta \neq 1 / 2$. If $\delta=1 / 2$, it is second order accuracy in the case $\theta \neq 1 / 12$ and forth order accuracy is achieved if $\theta=1 / 12$.

Only the first $m$ modes will be considered. We denote by

$$
u_{m}^{n}\left(x_{1}\right)=\sum_{i=0}^{m-1} q_{i}^{n} \phi_{i}\left(x_{1}\right), \quad \dot{u}_{m}^{n}\left(x_{1}\right)=\sum_{i=0}^{m-1} \dot{q}_{i}^{n} \phi_{i}\left(x_{1}\right), \quad \ddot{u}_{m}^{n}\left(x_{1}\right)=\sum_{i=0}^{m-1} \ddot{q}_{i}^{n} \phi_{i}\left(x_{1}\right)
$$

the approximations of $u\left(x_{1}, t_{n}\right), \frac{\partial u}{\partial t}\left(x_{1}, t_{n}\right), \frac{\partial^{2} u}{\partial t^{2}}\left(x_{1}, t_{n}\right)$ respectively.

\section{Approximations of the unsteady Navier-Stokes equations IN A MOVING DOMAIN}

\subsection{Strong form of the unsteady Navier-Stokes equations}

Let $u:[0, L] \times[0, T] \rightarrow \mathbb{R}$ be the transverse displacement of a thin elastic wall. For each time instant $t \in[0, T]$, we assume that $u(\cdot, t):[0, L] \rightarrow \mathbb{R}$ is at least of class $\mathcal{C}^{1}$. We suppose that an admissible displacement verifies:

$$
\begin{aligned}
u(0, t)= & \frac{\partial u}{\partial x_{1}}(0, t)=0, u(L, t)=\frac{\partial u}{\partial x_{1}}(L, t)=0, \quad \forall t \in[0, T], \\
& 0<H+u\left(x_{1}, t\right), \quad \forall\left(x_{1}, t\right) \in[0, L] \times[0, T]
\end{aligned}
$$

where $H$ is a positive constant.

For each $t \in[0, T]$, we introduce the notations (see Fig. 1)

$$
\begin{aligned}
\Omega_{t}^{F} & =\left\{\left(x_{1}, x_{2}\right) \in \mathbb{R}^{2} ; x_{1} \in(0, L), 0<x_{2}<H+u\left(x_{1}, t\right)\right\} \\
\Gamma_{t} & =\left\{\left(x_{1}, x_{2}\right) \in \mathbb{R}^{2} ; x_{1} \in(0, L), x_{2}=H+u\left(x_{1}, t\right)\right\}
\end{aligned}
$$




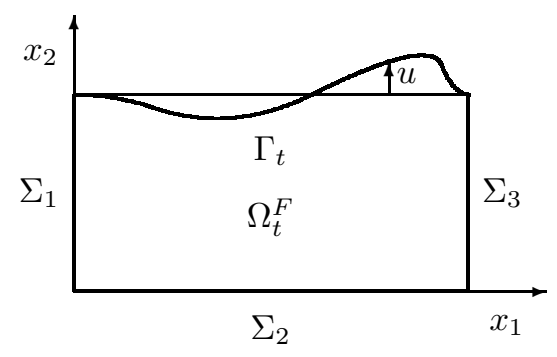

Figure 1. Example of an admissible domain.

Also, we denote

$$
\begin{aligned}
& \Sigma_{1}=\left\{\left(0, x_{2}\right) \in \mathbb{R}^{2} ; x_{2} \in(0, H)\right\} \\
& \Sigma_{2}=\left\{\left(x_{1}, 0\right) \in \mathbb{R}^{2} ; x_{1} \in(0, L)\right\} \\
& \Sigma_{3}=\left\{\left(L, x_{2}\right) \in \mathbb{R}^{2} ; x_{2} \in(0, H)\right\} .
\end{aligned}
$$

The two-dimensional domain occupied by the fluid is $\Omega_{t}^{F}$, the elastic wall is $\Gamma_{t}$, the rigid one is $\Sigma_{2}$, while $\Sigma_{1}$ and $\Sigma_{3}$ represent the upstream and downstream sections, respectively. The boundaries $\Sigma_{1}$ and $\Sigma_{3}$ are artificial.

In the following, we denote by $\mathbf{n}=\left(n_{1}, n_{2}\right)^{T}$ the unit outward normal vector and by $\boldsymbol{\tau}=\left(\tau_{1}, \tau_{2}\right)^{T}=$ $\left(-n_{2}, n_{1}\right)^{T}$ the unit tangential vector to $\partial \Omega_{t}^{F}$.

For all $t \in[0, T]$ and for all $\mathbf{x}=\left(x_{1}, x_{2}\right)^{T} \in \Omega_{t}^{F}$, find the velocity $\mathbf{v}(\mathbf{x}, t) \in \mathbb{R}^{2}$ and the pressure $p(\mathbf{x}, t) \in \mathbb{R}$ such that:

$$
\begin{aligned}
\rho^{F}\left(\frac{\partial \mathbf{v}}{\partial t}+(\mathbf{v} \cdot \nabla) \mathbf{v}\right)-\mu \Delta \mathbf{v}+\nabla p & =\mathbf{f}^{F}, \quad \forall t \in(0, T), \forall \mathbf{x} \in \Omega_{t}^{F} \\
\nabla \cdot \mathbf{v}= & 0, \quad \forall t \in(0, T), \forall \mathbf{x} \in \Omega_{t}^{F} \\
\mathbf{v} \times \mathbf{n}= & 0, \quad \text { on } \Sigma_{1} \times(0, T) \\
p= & P_{\text {in }}, \quad \text { on } \Sigma_{1} \times(0, T) \\
\mathbf{v}= & \mathbf{g}, \quad \text { on } \Sigma_{2} \times(0, T) \\
\mathbf{v} \times \mathbf{n}= & 0, \quad \text { on } \Sigma_{3} \times(0, T) \\
p= & P_{\text {out }}, \quad \text { on } \Sigma_{3} \times(0, T) \\
\mathbf{v}\left(x_{1}, H+u\left(x_{1}, t\right), t\right)= & \left(0, \frac{\partial u}{\partial t}\left(x_{1}, t\right)\right)^{T} \\
& \forall\left(x_{1}, t\right) \in(0, L) \times(0, T) \\
\mathbf{v}(\mathbf{x}, 0)= & \mathbf{v}^{0}(\mathbf{x}), \quad \forall \mathbf{x} \in \Omega_{0}^{F}
\end{aligned}
$$

where

- $\rho^{F}>0$ and $\mu>0$ are the mass density and the viscosity of the fluid;

- $\mathbf{f}^{F}=\left(f_{1}^{F}, f_{2}^{F}\right)$ are the applied volume forces, in general the gravity forces;

- $\mathbf{g}=\left(g_{1}, g_{2}\right)^{T}: \Sigma_{2} \times(0, T) \rightarrow \mathbb{R}^{2}$ is the imposed velocity profile on a part of the rigid boundary;

- $P_{\text {in }}: \Sigma_{1} \times(0, T) \rightarrow \mathbb{R}$ and $P_{\text {out }}: \Sigma_{3} \times(0, T) \rightarrow \mathbb{R}$ are prescribed boundary pressure;

- $\mathbf{v}^{0}: \Omega_{0}^{F} \rightarrow \mathbb{R}^{2}$ is initial velocity and $\Omega_{0}^{F}$ is the initial domain.

We have supposed that the displacement $u$ and the velocity $\frac{\partial u}{\partial t}$ of the moving wall are known, consequently the moving domain $\Omega_{t}^{F}$ which depends on $u$ and the prescribed velocity on the elastic boundary $\Gamma_{t}$ appearing in the boundary condition (2.24) are given. 
The following notations have been used: $\mathbf{v}=\left(v_{1}, v_{2}\right)^{T}, \nabla \cdot \mathbf{v}=\frac{\partial v_{1}}{\partial x_{1}}+\frac{\partial v_{2}}{\partial x_{2}}, \mathbf{v} \times \mathbf{n}=v_{1} n_{2}-v_{2} n_{1}, \Delta v_{i}=\frac{\partial^{2} v_{i}}{\partial x_{1}^{2}}+\frac{\partial^{2} v_{i}}{\partial x_{2}^{2}}$ for $i=1,2$,

$$
\nabla p=\left(\begin{array}{c}
\frac{\partial p}{\partial x_{1}} \\
\frac{\partial p}{\partial x_{2}}
\end{array}\right), \quad \Delta \mathbf{v}=\left(\begin{array}{c}
\Delta v_{1} \\
\Delta v_{2}
\end{array}\right), \quad(\mathbf{v} \cdot \nabla) \mathbf{v}=\left(\begin{array}{c}
v_{1} \frac{\partial v_{1}}{\partial x_{1}}+v_{2} \frac{\partial v_{1}}{\partial x_{2}} \\
v_{1} \frac{\partial v_{2}}{\partial x_{1}}+v_{2} \frac{\partial v_{2}}{\partial x_{2}}
\end{array}\right)
$$

The Navier-Stokes equations with boundary condition on pressure were firstly studied in [27].

\subsection{The Arbitrary Lagrangian Eulerian coordinates and the time discretization}

The Arbitrary Lagrangian Eulerian (ALE) framework was successfully used for the fluid structure interaction problems (see [28] and the references given there).

We denote by $\widehat{\Omega}^{F}=(0, L) \times(0, H)$ the reference domain and by $\widehat{\Gamma}=(0, L) \times\{H\}$ its top boundary. Since the moving boundary is a graph of a real function and the reference domain is a rectangle, we can construct explicitly so called the ALE map.

For each admissible displacement $u$, we consider following family of one-to-one continuous differentiable transformation $\mathcal{A}_{t}: \widehat{\Omega}^{F} \rightarrow \Omega_{t}^{F}$ given by:

$$
\mathcal{A}_{t}\left(\widehat{x}_{1}, \widehat{x}_{2}\right)=\left(\widehat{x}_{1}, \frac{H+u\left(\widehat{x}_{1}, t\right)}{H} \widehat{x}_{2}\right)^{T}
$$

which admits the continuous differentiable inverse mapping

$$
\mathcal{A}_{t}^{-1}\left(x_{1}, x_{2}\right)=\left(x_{1}, \frac{H x_{2}}{H+u\left(x_{1}, t\right)}\right)^{T}
$$

and verifies that $\mathcal{A}_{t}\left(\widehat{\Omega}^{F}\right)=\Omega_{t}^{F}, \mathcal{A}_{t}(\widehat{\Gamma})=\Gamma_{t}$ and $\mathcal{A}_{t}(\widehat{x})=\widehat{x}, \forall \widehat{x} \in \Sigma$.

We set $\mathbf{x}=\mathcal{A}_{t}(\widehat{\mathbf{x}})$ for each $\mathbf{x}=\left(x_{1}, x_{2}\right) \in \Omega_{t}^{F}$ and $\widehat{\mathbf{x}}=\left(\widehat{x}_{1}, \widehat{x}_{2}\right) \in \widehat{\Omega}^{F}$.

We denote by $\widehat{\mathbf{v}}(\widehat{\mathbf{x}}, t)=\mathbf{v}\left(\mathcal{A}_{t}(\widehat{\mathbf{x}}), t\right)$ and $\widehat{p}(\widehat{\mathbf{x}}, t)=p\left(\mathcal{A}_{t}(\widehat{\mathbf{x}}), t\right)$ the velocity and the pressure using so-called Arbitrary Lagrangian Eulerian coordinates.

Let $\widehat{\mathbf{x}}$ be fixed. According to the chain rule, we have

$$
\frac{\partial \widehat{\mathbf{v}}}{\partial t}(\widehat{\mathbf{x}}, t)=\frac{\mathrm{d}}{\mathrm{d} t}\left[\mathbf{v}\left(\mathcal{A}_{t}(\widehat{\mathbf{x}}), t\right)\right]=\left(\frac{\partial \mathcal{A}_{t}}{\partial t}(\widehat{\mathbf{x}}) \cdot \nabla\right) \mathbf{v}\left(\mathcal{A}_{t}(\widehat{\mathbf{x}}), t\right)+\frac{\partial \mathbf{v}}{\partial t}\left(\mathcal{A}_{t}(\widehat{\mathbf{x}}), t\right)
$$

which implies

$$
\frac{\partial \mathbf{v}}{\partial t}(\mathbf{x}, t)=\frac{\partial \widehat{\mathbf{v}}}{\partial t}(\widehat{\mathbf{x}}, t)-\left(\frac{\partial \mathcal{A}_{t}}{\partial t}(\widehat{\mathbf{x}}) \cdot \nabla\right) \mathbf{v}(\mathbf{x}, t) .
$$

Let $N \in \mathbb{N}^{*}$ be the number of time steps and $\Delta t=T / N$ the time step. We set $t_{n}=n \Delta t$ for $n=0,1, \ldots, N$. We will indicate $\mathbf{v}^{n+1}(\mathbf{x}), p^{n+1}(\mathbf{x})$ the approximations of $\mathbf{v}\left(\mathbf{x}, t_{n+1}\right), p\left(\mathbf{x}, t_{n+1}\right)$ for $\mathbf{x} \in \Omega_{t_{n+1}}^{F}$.

We denote $\mathbf{x}=\mathcal{A}_{t_{n+1}}(\widehat{\mathbf{x}})$ and consequently $\widehat{\mathbf{x}}=\mathcal{A}_{t_{n+1}}^{-1}(\mathbf{x})$.

We can use the first order finite difference scheme

$$
\begin{aligned}
\frac{\partial \widehat{\mathbf{v}}}{\partial t}\left(\widehat{\mathbf{x}}, t_{n+1}\right) \approx \frac{\widehat{\mathbf{v}}\left(\widehat{\mathbf{x}}, t_{n+1}\right)-\widehat{\mathbf{v}}\left(\widehat{\mathbf{x}}, t_{n}\right)}{\Delta t} & =\frac{\mathbf{v}\left(\mathcal{A}_{t_{n+1}}(\widehat{\mathbf{x}}), t_{n+1}\right)-\mathbf{v}\left(\mathcal{A}_{t_{n}}(\widehat{\mathbf{x}}), t_{n}\right)}{\Delta t} \\
& =\frac{\mathbf{v}\left(\mathbf{x}, t_{n+1}\right)-\mathbf{v}\left(\mathcal{A}_{t_{n}} \circ \mathcal{A}_{t_{n+1}}^{-1}(\mathbf{x}), t_{n}\right)}{\Delta t} \approx \frac{\mathbf{v}^{n+1}(\mathbf{x})-\mathbf{v}^{n}\left(\mathcal{A}_{t_{n}} \circ \mathcal{A}_{t_{n+1}}^{-1}(\mathbf{x})\right)}{\Delta t} .
\end{aligned}
$$

Observe that the derivative $\frac{\partial \widehat{\mathbf{v}}}{\partial t}\left(\widehat{\mathbf{x}}, t_{n+1}\right)$ which depends on the ALE coordinates $\widehat{\mathbf{x}}$ can be approached by the expression $\frac{\mathbf{v}^{n+1}(\mathbf{x})-\mathbf{v}^{n}\left(\mathcal{A}_{t_{n}} \circ \mathcal{A}_{t_{n+1}}^{-1}(\mathbf{x})\right)}{\Delta t}$ written using the Eulerian coordinates $\mathbf{x}$. 
By the definition, it follows

$$
\frac{\partial \mathcal{A}_{t}}{\partial t}(\widehat{\mathbf{x}})=\left(0, \frac{\frac{\partial u}{\partial t}\left(\widehat{x}_{1}, t\right)}{H} \widehat{x}_{2}\right)^{T}
$$

and by replacing $\widehat{\mathbf{x}}=\mathcal{A}_{t_{n+1}}^{-1}(\mathbf{x})$, we obtain

$$
\left.\boldsymbol{\vartheta}^{n+1}(\mathbf{x}) \stackrel{\text { def }}{=} \frac{\partial \mathcal{A}_{t}}{\partial t}(\widehat{\mathbf{x}})\right|_{t=t_{n+1}}=\left(0, \frac{\partial u}{\partial t}\left(x_{1}, t_{n+1}\right) \frac{x_{2}}{H+u\left(x_{1}, t_{n+1}\right)}\right)^{T}
$$

Finally, from the equality (2.26), we can employ the approximation

$$
\frac{\partial \mathbf{v}}{\partial t}\left(\mathbf{x}, t_{n+1}\right) \approx \frac{\mathbf{v}^{n+1}(\mathbf{x})-\mathbf{v}^{n}\left(\mathcal{A}_{t_{n}} \circ \mathcal{A}_{t_{n+1}}^{-1}(\mathbf{x})\right)}{\Delta t}-\left(\boldsymbol{\vartheta}^{n+1}(\mathbf{x}) \cdot \nabla\right) \mathbf{v}^{n+1}(\mathbf{x})
$$

The time-advancing scheme is: knowing the velocity $\mathbf{v}^{n}: \Omega_{t_{n}}^{F} \rightarrow \mathbb{R}^{2}$ of the fluid at the previous time step and the displacement $u\left(\cdot, t_{n+1}\right)$, the velocity $\frac{\partial u}{\partial t}\left(\cdot, t_{n+1}\right)$ of the moving boundary at the current time step, find the velocity $\mathbf{v}^{n+1}: \Omega_{t_{n+1}}^{F} \rightarrow \mathbb{R}^{2}$, the pressure $p^{n+1}: \Omega_{t_{n+1}}^{F} \rightarrow \mathbb{R}$ of the fluid, such that

$$
\begin{aligned}
\rho^{F}\left(\frac{\mathbf{v}^{n+1}}{\Delta t}+\left(\left(\mathbf{V}^{n}-\boldsymbol{\vartheta}^{n+1}\right) \cdot \nabla\right) \mathbf{v}^{n+1}\right)-\mu \Delta \mathbf{v}^{n+1}+\nabla p^{n+1} & =\rho^{F} \frac{\mathbf{V}^{n}}{\Delta t}+\mathbf{f}^{F} \text { in } \Omega_{t_{n+1}}^{F} \\
\nabla \cdot \mathbf{v}^{n+1} & =0 \text { in } \Omega_{t_{n+1}}^{F} \\
\mathbf{v}^{n+1} \times \mathbf{n}= & 0 \text { on } \Sigma_{1} \\
p^{n+1} & =P_{\text {in }}\left(\cdot, t_{n+1}\right) \text { on } \Sigma_{1} \\
\mathbf{v}^{n+1} & =\mathbf{g}\left(\cdot, t_{n+1}\right) \text { on } \Sigma_{2} \\
\mathbf{v}^{n+1} \times \mathbf{n}= & 0 \text { on } \Sigma_{3} \\
p^{n+1} & =P_{\text {out }}\left(\cdot, t_{n+1}\right) \text { on } \Sigma_{3} \\
\mathbf{v}^{n+1}\left(x_{1}, H+u\left(x_{1}, t_{n+1}\right), t\right) & \left(0, \frac{\partial u}{\partial t}\left(x_{1}, t_{n+1}\right)\right)^{T}, \\
& 0<x_{1}<L
\end{aligned}
$$

where $\mathbf{V}^{n}(\mathbf{x})=\mathbf{v}^{n}\left(\mathcal{A}_{t_{n}} \circ \mathcal{A}_{t_{n+1}}^{-1}(\mathbf{x})\right)$ for all $\mathbf{x}$ in $\Omega_{t_{n+1}}^{F}$.

This is a first order time accurate scheme. The time derivative was approached by the backward Euler Method. The nonlinear term $(\mathbf{v} \cdot \nabla) \mathbf{v}$ was treated semi-implicit, therefore we obtain a linear system whose associated matrix is not symmetric and it changes at each time step.

\subsection{Mixed finite element approximation}

We introduce the following Hilbert spaces:

$$
\begin{aligned}
W^{n+1} & =\left\{\mathbf{w} \in\left(H^{1}\left(\Omega_{t_{n+1}}^{F}\right)\right)^{2} ; \mathbf{w} \times \mathbf{n}=0 \text { on } \Sigma_{1} \cup \Sigma_{3}, \mathbf{w}=0 \text { on } \Sigma_{2} \cup \Gamma_{t_{n+1}}\right\}, \\
Q^{n+1} & =L^{2}\left(\Omega_{t_{n+1}}^{F}\right) .
\end{aligned}
$$


Find the velocity $\mathbf{v}^{n+1} \in\left(H^{1}\left(\Omega_{t_{n+1}}^{F}\right)\right)^{2}$ satisfies the boundary conditions (2.30), (2.32), (2.33), (2.35) and the pressure $p^{n+1} \in L^{2}\left(\Omega_{t_{n+1}}^{F}\right)$ such that

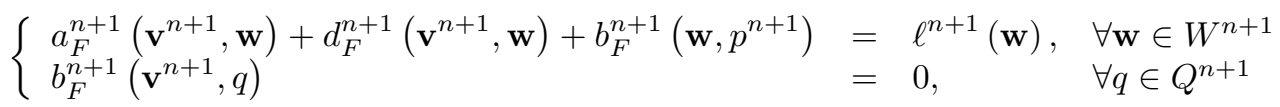

where

$$
\begin{aligned}
a_{F}^{n+1}\left(\mathbf{v}^{n+1}, \mathbf{w}\right)= & \frac{\rho^{F}}{\Delta t}\left(\mathbf{v}^{n+1}, \mathbf{w}\right) \\
& +\mu\left(\nabla \times \mathbf{v}^{n+1}, \nabla \times \mathbf{w}\right)+\mu\left(\nabla \cdot \mathbf{v}^{n+1}, \nabla \cdot \mathbf{w}\right) \\
d_{F}^{n+1}\left(\mathbf{v}^{n+1}, \mathbf{w}\right)= & \rho^{F}\left(\left(\left(\mathbf{V}^{n}-\vartheta^{n+1}\right) \cdot \nabla\right) \mathbf{v}^{n+1}, \mathbf{w}\right) \\
b_{F}^{n+1}(\mathbf{w}, q)= & -(\nabla \cdot \mathbf{w}, q) \\
\ell^{n+1}(\mathbf{w})= & \frac{\rho^{F}}{\Delta t}\left(\mathbf{V}^{n}, \mathbf{w}\right)+\left(\mathbf{f}^{F}, \mathbf{w}\right) \\
& -\int_{\Sigma_{1}} P_{\text {in }}\left(\cdot, t_{n+1}\right) \mathbf{n} \cdot \mathbf{w} \mathrm{d} \gamma-\int_{\Sigma_{3}} P_{\text {out }}\left(\cdot, t_{n+1}\right) \mathbf{n} \cdot \mathbf{w} \mathrm{d} \gamma
\end{aligned}
$$

and $(\cdot, \cdot)$ is the scalar product of $L^{2}\left(\Omega_{t_{n+1}}^{F}\right)$ or $\left(L^{2}\left(\Omega_{t_{n+1}}^{F}\right)\right)^{2}$.

Following [17, 27], the bilinear form $(\nabla \times \mathbf{y}, \nabla \times \mathbf{w})+(\nabla \cdot \mathbf{y}, \nabla \cdot \mathbf{w})$ is $W^{n+1}$ elliptic and $b_{F}^{n+1}$ satisfies infsup condition or Ladyzhenskaya-Babuska-Brezzi condition. If the bilinear form $a_{F}^{n+1}+d_{F}^{n+1}$ is elliptic on the subspace $\left\{\mathbf{w} \in W^{n+1} ; \nabla \cdot \mathbf{w}=0\right\}$, then the problem (2.36) has an unique solution.

For the approximation of the fluid velocity we have been used the finite elements $\mathbb{P}_{1}+$ bubble also refereed to as MINI elements introduced by Arnold, Brezzi and Fortin. For the fluid pressure the finite elements $\mathbb{P}_{1}$ have been employed.

\section{Approximation of the COUPled Fluid-Structure equations}

\subsection{Strong form of the coupled equations}

In the previous sections, we have introduced separately the structure and the fluid equations.

The coupled fluid structure problem is: find the transverse displacement $u$ satisfies (1.1)-(1.5), the velocity $\mathbf{v}$ and the pressure $p$ satisfy $(2.17)-(2.25)$ such that

$$
\eta\left(x_{1}, t\right)=-\left(\sigma^{F} \mathbf{n} \cdot \mathbf{e}_{2}\right)_{\left(x_{1}, H+u\left(x_{1}, t\right)\right)} \sqrt{1+\left(\frac{\partial u}{\partial x_{1}}\left(x_{1}, t\right)\right)^{2}}
$$

where $\sigma^{F}=-p I+\mu\left(\nabla \mathbf{v}+\nabla \mathbf{v}^{T}\right)$ is the stress tensor of the fluid, $\mathbf{e}_{2}=(0,1)^{T}$ is the unit vector in the $x_{2}$ direction.

The displacement of the structure depends on the vertical component of the stresses exerted by the fluid on the interface (Eqs. (1.1) and (3.41)). This cames from the continuity of the stresses across the interface.

The movement of the structure changes the domain where the fluid equations must be solved (Eqs. (2.17) and (2.18)). Also, on the interface we have to impose the equality between the fluid and structure velocity (Eq. (2.24)).

The stresses exerted by the fluid $-\sigma^{F} n$ are defined on the elastic wall $\Gamma_{t}$, while the stresses on the structure $\eta$ are defined on the horizontal segment $\widehat{\Gamma}$. The factor $\sqrt{1+\left(\frac{\partial u}{\partial x_{1}}\left(x_{1}, t\right)\right)^{2}}$ which appears in the equation (3.41) 
is necessary to have

$$
\int_{\Gamma_{t}} \sigma^{F} \mathbf{n} \cdot \mathbf{e}_{2} \mathrm{~d} \gamma=\int_{0}^{L}\left(\sigma^{F} \mathbf{n} \cdot \mathbf{e}_{2}\right)_{\left(x_{1}, H+u\left(x_{1}, t\right)\right)} \sqrt{1+\left(\frac{\partial u}{\partial x_{1}}\left(x_{1}, t\right)\right)^{2}} \mathrm{~d} x_{1}
$$

The displacement $u$ must be admissible or equivalent the elastic wall $\Gamma_{t}$ does not touch the bottom boundary $\Sigma_{2}$.

The existence results for the fluid structure interaction can be found for example in $[1,15]$ for the steady case and in $[2,4,9,16]$ for the unsteady case. We didn't cited here the results concerning the interaction between a fluid and a rigid solid in rotation or in translation.

\subsection{Identification of the stresses on the interface using the Least Squares Method}

We recall that, the most frequently, the fluid-structure interaction problems are solved numerically by partitioned procedures, i.e. the fluid and the structure equations are solved separately. This can be done by using fixed point or Newton like methods. If the starting point is not chosen "sufficiently close" to the solution, these methods diverge.

In the following, the equation (3.41) will be treated by the Least Squares Method and at each time step we have to solve an optimization problem which is less sensitive to the choice of the starting point. This is the main advantage of this approach.

In order to evaluate the cost function, we must call one time the structure solver, to update the mesh and to call one time the fluid solver. We present the details below.

The unknowns of the optimization problem are the stresses on the interface.

Suppose that at the previous time step $t_{n}$ we know:

- the approximations of the displacement, the velocity and the acceleration of the structure denoted respectively by

$$
u_{m}^{n}\left(x_{1}\right)=\sum_{i=0}^{m-1} q_{i}^{n} \phi_{i}\left(x_{1}\right), \quad \dot{u}_{m}^{n}\left(x_{1}\right)=\sum_{i=0}^{m-1} \dot{q}_{i}^{n} \phi_{i}\left(x_{1}\right), \quad \ddot{u}_{m}^{n}\left(x_{1}\right)=\sum_{i=0}^{m-1} \ddot{q}_{i}^{n} \phi_{i}\left(x_{1}\right)
$$

- the polygonal approximation of the fluid domain denoted by $\Omega_{h}^{F, n}$;

- the finite element approximations of the velocity and the pressure of the fluid denoted by $\mathbf{v}_{h}^{n}$ and $p_{h}^{n}$ respectively.

We seek an approximation of the stresses on the interface at the current time step $t_{n+1}$ of the form $\eta_{m}^{n+1}\left(x_{1}\right)=$ $\sum_{i=0}^{m-1} \alpha_{i}^{n+1} \phi_{i}\left(x_{1}\right)$, where $\alpha_{i}^{n+1}, i=0, \ldots, m-1$ are the parameters to be identified.

Let $\boldsymbol{\alpha}=\left(\alpha_{0}, \ldots, \alpha_{m-1}\right) \in \mathbb{R}^{m}$.

\section{Structure sub-problem}

For $i=0, \ldots, m-1$, knowing $q_{i}^{n}, \dot{q}_{i}^{n}, \ddot{q}_{i}^{n}$, find $Q_{i}, \dot{Q}_{i}, \ddot{Q}_{i}$ such that:

$$
\begin{aligned}
& \left(1+\omega_{i}^{2}(\Delta t)^{2} \theta\right) \ddot{Q}_{i}=\frac{1}{\rho^{S} h^{S}} \alpha_{i}-\omega_{i}^{2}\left[q_{i}^{n}+\Delta t \dot{q}_{i}^{n}+(\Delta t)^{2}\left(\frac{1}{2}-\theta\right) \ddot{q}_{i}^{n}\right] \\
& \dot{Q}_{i}=\dot{q}_{i}^{n}+\Delta t\left[(1-\delta) \ddot{q}_{i}^{n}+\delta \ddot{Q}_{i}\right] \\
& Q_{i}=q_{i}^{n}+\Delta t \dot{q}_{i}^{n}+(\Delta t)^{2}\left[\left(\frac{1}{2}-\theta\right) \ddot{q}_{i}^{n}+\theta \ddot{Q}_{i}\right]
\end{aligned}
$$

The above equations have been obtained from (1.16), (1.13), (1.14) replacing $\alpha_{i}^{n+1}, q_{i}^{n+1}, \dot{q}_{i}^{n+1}, \ddot{q}_{i}^{n+1}$ by $\alpha_{i}, Q_{i}$, $\dot{Q}_{i}, \ddot{Q}_{i}$ respectively. 
Set

$$
U\left(x_{1}\right)=\sum_{i=0}^{m-1} Q_{i} \phi_{i}\left(x_{1}\right), \quad \dot{U}\left(x_{1}\right)=\sum_{i=0}^{m-1} \dot{Q}_{i} \phi_{i}\left(x_{1}\right), \quad \ddot{U}\left(x_{1}\right)=\sum_{i=0}^{m-1} \ddot{Q}_{i} \phi_{i}\left(x_{1}\right) .
$$

\section{Fluid sub-problem}

Let $\widehat{\mathcal{T}}_{h}$ be a mesh with triangular elements of the reference domain $\widehat{\Omega}^{F}$. We define the mesh with triangular elements $\mathcal{T}_{h}$ by moving each node of $\widehat{\mathcal{T}}_{h}$ using the map

$$
\mathcal{A}_{U}\left(\widehat{x}_{1}, \widehat{x}_{2}\right)=\left(\widehat{x}_{1}, \frac{H+U\left(\widehat{x}_{1}\right)}{H} \widehat{x}_{2}\right)^{T} \text {. }
$$

We denote by $\Omega_{h}^{F}$ the polygonal domain corresponding to the mesh $\mathcal{T}_{h}$ and we have $\partial \Omega_{h}^{F}=\Sigma_{1} \cup \Sigma_{2} \cup \Sigma_{3} \cup \Gamma_{h}$ where $\Gamma_{h}$ is the top boundary.

Let us introduce the finite dimension spaces

$$
\begin{aligned}
W_{h}= & \left\{\mathbf{w}_{h} \in\left(C^{0}\left(\bar{\Omega}_{h}^{F}\right)\right)^{2} ; \forall K \text { triangle of } \mathcal{T}_{h},\left.\mathbf{w}_{h}\right|_{K} \in P 1+\text { bubble },\right. \\
& \left.\mathbf{w}_{h} \times \mathbf{n}=0 \text { on } \Sigma_{1} \cup \Sigma_{3}, \mathbf{w}_{h}=0 \text { on } \Sigma_{2} \cup \Gamma_{h}\right\} \\
Q_{h}= & \left\{q_{h} \in C^{0}\left(\bar{\Omega}_{h}^{F}\right) ; \forall K \text { triangle of } \mathcal{T}_{h},\left.q_{h}\right|_{K} \in P 1\right\} .
\end{aligned}
$$

Remark that the finite element spaces are defined directly on the physical domain. Many authors (see $[22,28,29]$ ) use a different framework: the test function in the physical domain is obtained from the one in a reference domain via the ALE map. In this case, we have to pay attention to the quadrature formulas (see [28]).

Find the velocity $\mathbf{v}_{h}$ satisfies the boundary conditions

$$
\begin{aligned}
\mathbf{v}_{h} \times \mathbf{n} & =0, \text { on each vertex of } \Sigma_{1} \cup \Sigma_{3}, \\
\mathbf{v}_{h} & =\mathbf{g}\left(\cdot, t_{n+1}\right), \text { on each vertex of } \Sigma_{2}, \\
\mathbf{v}_{h} & =(0, \dot{U})^{T}, \text { on each vertex of the top boundary } \Gamma_{h}
\end{aligned}
$$

and the pressure $p_{h} \in Q_{h}$ such that

$$
\left\{\begin{array}{llll}
a_{F}^{n+1}\left(\mathbf{v}_{h}, \mathbf{w}_{h}\right)+d_{F}^{n+1}\left(\mathbf{v}_{h}, \mathbf{w}_{h}\right)+b_{F}^{n+1}\left(\mathbf{w}_{h}, p_{h}\right) & =\ell^{n+1}\left(\mathbf{w}_{h}\right), & & \forall \mathbf{w}_{h} \in W_{h} \\
b_{F}^{n+1}\left(\mathbf{v}_{h}, q_{h}\right) & =0, & & \forall q_{h} \in Q_{h} .
\end{array}\right.
$$

When $\ell^{n+1}\left(\mathbf{w}_{h}\right)$ is evaluated, we have to replace $\mathcal{A}_{t_{n}} \circ \mathcal{A}_{t_{n+1}}^{-1}$ by $\mathcal{A}_{u_{m}^{n}} \circ \mathcal{A}_{U}^{-1}$ in the definition (2.40).

\section{Definition of the cost function}

The right side part of the equation (3.41), which represents the stresses from fluid acting on the interface, will be approached by $\sum_{i=0}^{m-1} \beta_{i} \phi_{i}\left(x_{1}\right)$.

Let us define for $i=0, \ldots, m-1$

$$
\beta_{i}=-\int_{0}^{L} \phi_{i}\left(x_{1}\right)\left(\sigma^{F}\left(\mathbf{v}_{h}, p_{h}\right) \mathbf{n} \cdot \mathbf{e}_{2}\right)_{\left(x_{1}, H+U\left(x_{1}\right)\right)} \sqrt{1+\left(\frac{\partial U}{\partial x_{1}}\left(x_{1}\right)\right)^{2}} \mathrm{~d} x_{1} .
$$

Since $\mathbf{n}=\left(n_{1}, n_{2}\right)^{T}=\frac{1}{\sqrt{1+\left(\frac{\partial U}{\partial x_{1}}\right)^{2}}}\left(-\frac{\partial U}{\partial x_{1}}, 1\right)^{T}$, we obtain

$$
\beta_{i}=\int_{0}^{L} \phi_{i}\left(x_{1}\right)\left(p_{h}-\mu\left(\frac{\partial v_{h, 1}}{\partial x_{2}}+\frac{\partial v_{h, 2}}{\partial x_{1}}\right)\left(-\frac{\partial U}{\partial x_{1}}\right)-2 \mu \frac{\partial v_{h, 2}}{\partial x_{2}}\right)_{\left(x_{1}, H+U\left(x_{1}\right)\right)} \mathrm{d} x_{1} .
$$


Set the cost function

$$
J^{n+1}(\boldsymbol{\alpha})=\frac{1}{2} \sum_{i=0}^{m-1}\left(\alpha_{i}-\beta_{i}\right)^{2} .
$$

The terms containing the viscosity can be neglected from the boundary expressions. Consequently, we could use the simpler formula

$$
\beta_{i}=\int_{0}^{L} \phi_{i}\left(x_{1}\right) p_{h}\left(x_{1}, H+U\left(x_{1}\right)\right) \mathrm{d} x_{1}, \quad i=0, \ldots, m-1 .
$$

We recall that the stresses on the interface at the current time step $t_{n+1}$ will be approached by $\eta_{m}^{n+1}\left(x_{1}\right)=$ $\sum_{i=0}^{m-1} \alpha_{i}^{n+1} \phi_{i}\left(x_{1}\right)$.

The parameters $\alpha_{i}^{n+1}$ for $0 \leq i \leq m-1$ will be "identified" solving an optimization problem, more precisely

$$
\boldsymbol{\alpha}^{n+1} \stackrel{\text { def }}{=}\left(\alpha_{0}^{n+1}, \ldots, \alpha_{m-1}^{n+1}\right) \in \arg \min _{\boldsymbol{\alpha} \in \mathbb{R}^{m}} J^{n+1}(\boldsymbol{\alpha}) .
$$

We will see in the following that the above defined cost function is related to the fixed point approach for fluid-structure interaction.

We introduce the structure operator given by $\mathcal{S}(\boldsymbol{\alpha})=(Q, \dot{Q}, \ddot{Q})$ and the fluid operator given by $\mathcal{F}(Q, \dot{Q}, \ddot{Q})=\boldsymbol{\beta}$, where $(Q, \dot{Q}, \ddot{Q})=\left(Q_{i}, \dot{Q}_{i}, \ddot{Q}_{i}\right)_{0 \leq i \leq m-1}$.

Our approach is to minimize

$$
J^{n+1}(\boldsymbol{\alpha})=\frac{1}{2}\|\boldsymbol{\alpha}-\boldsymbol{\beta}\|_{2}^{2}=\frac{1}{2}\|\boldsymbol{\alpha}-\mathcal{F} \circ \mathcal{S}(\boldsymbol{\alpha})\|_{2}^{2}
$$

where $\|\cdot\|_{2}$ stands the Euclidean norm of $\mathbb{R}^{m}$.

The fixed point framework is to solve $\mathcal{F} \circ \mathcal{S}(\boldsymbol{\alpha})=\boldsymbol{\alpha}$.

Some authors use the displacement of the structure in place of $\boldsymbol{\alpha}$ as a fixed point. In the following we will study the sensitivity of the displacement and velocity of the structure with respect to $\boldsymbol{\alpha}$. This enables us to compare the solution computed minimizing the cost function with the ones presented in $[12,14,26]$.

Let

$$
(\mathrm{d} Q, \mathrm{~d} \dot{Q}, \mathrm{~d} \ddot{Q})=\mathcal{S}(\boldsymbol{\alpha})-\mathcal{S}(\boldsymbol{\beta})
$$

where $(\mathrm{d} Q, \mathrm{~d} \dot{Q}, \mathrm{~d} \ddot{Q})=\left(\mathrm{d} Q_{i}, \mathrm{~d} \dot{Q}_{i}, \mathrm{~d} \ddot{Q}_{i}\right)_{0 \leq i \leq m-1}$ and set

$$
\mathrm{d} U\left(x_{1}\right)=\sum_{i=0}^{m-1} \mathrm{~d} Q_{i} \phi_{i}\left(x_{1}\right), \quad \mathrm{d} \dot{U}\left(x_{1}\right)=\sum_{i=0}^{m-1} \mathrm{~d} \dot{Q}_{i} \phi_{i}\left(x_{1}\right) .
$$

We denote by $\|\cdot\|_{L^{2}(0, L)}$ the usual norm of the space $L^{2}(0, L)$.

Proposition 3.1. We have

$$
\begin{aligned}
\|\mathrm{d} U\|_{L^{2}(0, L)} & \leq \frac{(\Delta t)^{2} \theta}{\rho^{S} h^{S}} \sqrt{2 J^{n+1}(\boldsymbol{\alpha})} \\
\|\mathrm{d} \dot{U}\|_{L^{2}(0, L)} & \leq \frac{(\Delta t) \delta}{\rho^{S} h^{S}} \sqrt{2 J^{n+1}(\boldsymbol{\alpha})}
\end{aligned}
$$


Proof. From the equations (3.42)-(3.44), we obtain that

$$
\mathrm{d} \ddot{Q}_{i}=\frac{1}{\left(1+\omega_{i}^{2}(\Delta t)^{2} \theta\right)} \frac{1}{\rho^{S} h^{S}}\left(\alpha_{i}-\beta_{i}\right), \quad \mathrm{d} \dot{Q}_{i}=(\Delta t) \delta \mathrm{d} \ddot{Q}_{i}, \quad \mathrm{~d} Q_{i}=(\Delta t)^{2} \theta \mathrm{d} \ddot{Q}_{i} .
$$

Since $\theta>0$, we have $\left(1+\omega_{i}^{2}(\Delta t)^{2} \theta\right)>1$ and consequently

$$
\left|\mathrm{d} \ddot{Q}_{i}\right| \leq \frac{1}{\rho^{S} h^{S}}\left|\alpha_{i}-\beta_{i}\right|, \quad\left|\mathrm{d} \dot{Q}_{i}\right| \leq \frac{(\Delta t) \delta}{\rho^{S} h^{S}}\left|\alpha_{i}-\beta_{i}\right|, \quad\left|\mathrm{d} Q_{i}\right| \leq \frac{(\Delta t)^{2} \theta}{\rho^{S} h^{S}}\left|\alpha_{i}-\beta_{i}\right| .
$$

Using that $\left\{\phi_{i}\right\}_{i \in \mathbb{N}}$ is a orthonormal basis of $L^{2}(0, L)$, we have

$$
\|\mathrm{d} U\|_{L^{2}(0, L)}^{2}=\sum_{i=0}^{m-1}\left|\mathrm{~d} Q_{i}\right|^{2} \leq\left(\frac{(\Delta t)^{2} \theta}{\rho^{S} h^{S}}\right)^{2} \cdot \sum_{i=0}^{m-1}\left|\alpha_{i}-\beta_{i}\right|^{2} .
$$

By definition $2 J^{n+1}(\boldsymbol{\alpha})=\sum_{i=0}^{m-1}\left|\alpha_{i}-\beta_{i}\right|^{2}$. Therefore, it follows (3.46).

In the same manner, we can prove (3.47).

The inequalities (3.46) and (3.47) mean that the difference between the displacements or the velocities of the structure obtained at two consecutive steps of fixed point algorithm is bounded by an expression depending on the cost function.

\subsection{Coupled fluid-structure algorithm by the BFGS Method}

In order to solve at the current time step $t_{n+1}$ the optimization problem $\min J^{n+1}(\boldsymbol{\alpha})$ for $\boldsymbol{\alpha} \in \mathbb{R}^{m}$, we employ the quasi-Newton iterative Method called Broyden, Fletcher, Goldforb, Shano (BFGS) scheme (see for example [6], Chap. 9). This algorithm was employed in [24,25], but for a steady fluid-structure interaction.

Step 0. Choose a starting point $\boldsymbol{\alpha}^{n+1,0} \in \mathbb{R}^{m}$, an $m \times m$ symmetric positive matrix $H_{0}$ and a positive scalar $\epsilon$. Set $k=0$.

Step 1. Compute $\nabla J^{n+1}\left(\boldsymbol{\alpha}^{n+1, k}\right)$.

Step 2. If $\left\|\nabla J^{n+1}\left(\boldsymbol{\alpha}^{n+1, k}\right)\right\|<\epsilon$ stop.

Step 3. Set $\mathbf{d}^{k}=-H_{k} \nabla J^{n+1}\left(\boldsymbol{\alpha}^{n+1, k}\right)$.

Step 4. Determine $\boldsymbol{\alpha}^{n+1, k+1}=\boldsymbol{\alpha}^{n+1, k}+\theta_{k} \mathbf{d}^{k}, \theta_{k}>0$ by means of an approximate minimization

$$
J^{n+1}\left(\boldsymbol{\alpha}^{n+1, k+1}\right) \approx \min _{\theta \geq 0} J^{n+1}\left(\boldsymbol{\alpha}^{n+1, k}+\theta \mathbf{d}^{k}\right) .
$$

Step 5. Compute $\delta_{k}=\boldsymbol{\alpha}^{n+1, k+1}-\boldsymbol{\alpha}^{n+1, k}$.

Step 6. Compute $\nabla J^{n+1}\left(\boldsymbol{\alpha}^{n+1, k+1}\right)$ and $\gamma_{k}=\nabla J^{n+1}\left(\boldsymbol{\alpha}^{n+1, k+1}\right)-\nabla J^{n+1}\left(\boldsymbol{\alpha}^{n+1, k}\right)$.

Step 7. Compute

$$
H_{k+1}=H_{k}+\left(1+\frac{\gamma_{k}^{T} H_{k} \gamma_{k}}{\delta_{k}^{T} \gamma_{k}}\right) \frac{\delta_{k} \delta_{k}^{T}}{\delta_{k}^{T} \gamma_{k}}-\frac{\delta_{k} \gamma_{k}^{T} H_{k}+H_{k} \gamma_{k} \delta_{k}^{T}}{\delta_{k}^{T} \gamma_{k}}
$$

Step 8. Update $k=k+1$ and go to the Step 2.

The matrices $H_{k}$ approach the inverse of the Hessian of $J$.

For the inaccurate line search at the Step 4, the methods of Goldstein and Armijo were used.

The coupled fluid-structure algorithm is: suppose that at the previous time step $t_{n}$ we know $u_{m}^{n}=\sum_{i=0}^{m-1} q_{i}^{n} \phi_{i}$, $\dot{u}_{m}^{n}=\sum_{i=0}^{m-1} \dot{q}_{i}^{n} \phi_{i}, \ddot{u}_{m}^{n}=\sum_{i=0}^{m-1} \ddot{q}_{i}^{n} \phi_{i}, \mathbf{v}_{h}^{n}$ and $p_{h}^{n}$, then solve

$$
\boldsymbol{\alpha}^{n+1} \in \arg \min _{\boldsymbol{\alpha} \in \mathbb{R}^{m}} J^{n+1}(\boldsymbol{\alpha})
$$


using the BFGS scheme. The solution $\boldsymbol{\alpha}^{n+1}$ is the last term of the suite $\boldsymbol{\alpha}^{n+1,0}, \ldots, \boldsymbol{\alpha}^{n+1, k}, \boldsymbol{\alpha}^{n+1, k+1}, \ldots$

The stresses on the interface at the current time step $t_{n+1}$ are given by

$$
\eta_{m}^{n+1}\left(x_{1}\right)=\sum_{i=0}^{m-1} \alpha_{i}^{n+1} \phi_{i}\left(x_{1}\right)
$$

At each evaluation of the cost function we have to solve one structure sub-problem, to update the mesh and to solve one fluid sub-problem. We denote by $u_{m}^{n+1}, \dot{u}_{m}^{n+1}, \ddot{u}_{m}^{n+1}$ the solution of the structure sub-problem and by $\mathbf{v}_{h}^{n+1}$ and $p_{h}^{n+1}$ the solution of the fluid sub-problem when $J^{n+1}$ is evaluated in the point $\boldsymbol{\alpha}^{n+1}$.

In this paper, we compute $\nabla J^{n+1}(\boldsymbol{\alpha})$ by the Finite Differences Method

$$
\frac{\partial J^{n+1}}{\partial \alpha_{k}}(\boldsymbol{\alpha}) \approx \frac{J^{n+1}\left(\boldsymbol{\alpha}+\Delta \alpha_{k} \mathbf{e}_{\mathbf{k}}\right)-J^{n+1}(\boldsymbol{\alpha})}{\Delta \alpha_{k}}
$$

where $\mathbf{e}_{\mathbf{k}}$ is the $k$-th vector of the canonical base of $\mathbb{R}^{m}$ and $\Delta \alpha_{k}>0$ is the grid spacing.

\subsection{Fixed point, Newton and BFGS Methods}

In this section, we analyze different iterative methods for solving coupled fluid-structure problems.

In the previous section, we have presented the BFGS Method in order to solve the optimization problem

$$
\inf _{\boldsymbol{\alpha} \in \mathbb{R}^{m}} J(\boldsymbol{\alpha})=\frac{1}{2}\|\boldsymbol{\alpha}-\mathcal{F} \circ \mathcal{S}(\boldsymbol{\alpha})\|^{2}
$$

where $\|\cdot\|$ stands the Euclidean norm of $\mathbb{R}^{m}$.

The fixed point framework is to solve $\mathcal{F} \circ \mathcal{S}(\boldsymbol{\alpha})=\boldsymbol{\alpha}$.

Let $G: \mathbb{R}^{m} \rightarrow \mathbb{R}^{m}$ the nonlinear application given by $G(\boldsymbol{\alpha})=\mathcal{F} \circ \mathcal{S}(\boldsymbol{\alpha})$. In order to approach the fixed point $G\left(\boldsymbol{\alpha}^{*}\right)=\boldsymbol{\alpha}^{*}$, we could use the following algorithm

$$
\boldsymbol{\alpha}^{0} \in \mathbb{R}^{n}, \quad \boldsymbol{\alpha}^{k+1}=G\left(\boldsymbol{\alpha}^{k}\right) .
$$

If $G$ is a contraction and if the starting point $\boldsymbol{\alpha}^{0}$ is sufficiently close to the solution, then the sequence $\left\{\boldsymbol{\alpha}^{k}\right\}$ is linearly convergent to $\boldsymbol{\alpha}^{*}$.

We can set $F: \mathbb{R}^{m} \rightarrow \mathbb{R}^{m}, F(\boldsymbol{\alpha})=\boldsymbol{\alpha}-G(\boldsymbol{\alpha})$, then the fixed point problem is equivalent to $F(\boldsymbol{\alpha})=0$. The Newton Method can be employed for finding the roots of $F$ :

$$
\boldsymbol{\alpha}^{0} \in \mathbb{R}^{m}, \quad \boldsymbol{\alpha}^{k+1}=\boldsymbol{\alpha}^{k}-\left(\nabla F\left(\boldsymbol{\alpha}^{k}\right)^{T}\right)^{-1} F\left(\boldsymbol{\alpha}^{k}\right) .
$$

If the Jacobian matrix $\nabla F\left(\boldsymbol{\alpha}^{*}\right)^{T}$ is nonsingular and the starting point is sufficiently close to the solution, then the sequence $\left\{\boldsymbol{\alpha}^{k}\right\}$ is quadratically convergent.

In a general framework, the BFGS Method is designed to find approximation of the local minimizers of $J$, solutions of the nonlinear system $\nabla J(\boldsymbol{\alpha})=0$. Its convergence is superlinearly. In our particular case $J(\boldsymbol{\alpha})=\frac{1}{2}\|F(\boldsymbol{\alpha})\|^{2}$, we get

$$
\nabla J(\boldsymbol{\alpha})=(\nabla F(\boldsymbol{\alpha})) F(\boldsymbol{\alpha})
$$

Consequently, if $\boldsymbol{\alpha}^{*}$ is a local minimizer, then $\left(\nabla F\left(\boldsymbol{\alpha}^{*}\right)\right) F\left(\boldsymbol{\alpha}^{*}\right)=0$. What is most surprising is the fact that if the Jacobian matrix $\nabla F\left(\boldsymbol{\alpha}^{*}\right)^{T}$ is nonsingular, from the above equality we obtain that $F\left(\boldsymbol{\alpha}^{*}\right)=0$ ! In other words, a local minimizer $\boldsymbol{\alpha}^{*}$, with nonsingular Jacobian matrix $\nabla F\left(\boldsymbol{\alpha}^{*}\right)^{T}$, is a global minimizer of zero residual, i.e. $J\left(\boldsymbol{\alpha}^{*}\right)=0$. Only in the case when $\nabla F\left(\boldsymbol{\alpha}^{*}\right)^{T}$ is singular and $F\left(\boldsymbol{\alpha}^{*}\right) \neq 0$, the solution computed by the BFGS Method is not a solution of the fluid-structure coupled problem.

We have to recall that the Newton Method fails if $\nabla F\left(\boldsymbol{\alpha}^{*}\right)^{T}$ is nonsingular. 
TABLE 1. Parameters for the three tests with same time step and different mesh sizes.

\begin{tabular}{|c|c|c|c|}
\hline$\Delta t$ & mesh size $h$ & no. triangles & no. vertices \\
\hline 0.0005 & $h_{1}=0.25$ & 196 & 127 \\
\hline 0.0005 & $h_{2}=0.17$ & 226 & 448 \\
\hline 0.0005 & $h_{3}=0.10$ & 1250 & 696 \\
\hline
\end{tabular}

Concerning the convergence rate, the fixed point algorithm is slower than the BFGS Method, which is slower than the Newton Method. But, if the starting point is not sufficiently close to the solution, the fixed point and Newton algorithms diverge.

On the contrary, the BFGS Method is less sensitive to the choice of the starting point and, in general, it is convergent to a local minimizer from almost any starting point. This is the main advantage.

At each iteration of the Newton Method we have to solve a linear system of matrix $\nabla F\left(\boldsymbol{\alpha}^{k}\right)^{T}$, but it is not the case if we employ the BFGS Method, since the matrices $H_{k}$ approach the inverse of the Hessian. Moreover, if the Jacobian matrix $\nabla F\left(\boldsymbol{\alpha}^{k}\right)^{T}$ is singular or ill-conditioned, the Newton Method doesn't work.

\section{Numerical ReSults}

\subsection{Case of an impulsive pressure wave in a higher compliant channel}

Input data. We have tested on the 2D benchmark proposed in [13] arising from blood flow in arteries. Our numerical experiments have only an academic purpose. The structure equation (1.1) is not appropriate to model the artery wall. However, the algorithm presented in this paper can be easily adapted to simulate the blood flow in arteries by replacing (1.1) by the one dimensional axisymmetric membrane model.

The computation has been made in a domain of length $L=6 \mathrm{~cm}$ and height $H=1 \mathrm{~cm}$. The viscosity of the fluid was taken to be $\mu=0.035 \frac{\mathrm{g}}{\mathrm{cm} \cdot \mathrm{s}}$, its density $\rho^{F}=1 \frac{\mathrm{g}}{\mathrm{cm}^{3}}$. The thickness of the elastic wall is $h^{S}=0.1 \mathrm{~cm}$, the Young modulus $E=0.75 \cdot 10^{6} \frac{\mathrm{g}}{\mathrm{cm} \cdot \mathrm{s}^{2}}$, the Poisson ratio $\nu=0.5$, the density $\rho^{S}=1.1 \frac{\mathrm{g}}{\mathrm{cm}^{3}}$. The volume force in fluid is $\mathbf{f}^{F}=(0,0)^{T}$.

We remark that the structure is light and its density is comparable to the ones of the fluid. For this data, the fixed point algorithm with relaxation can diverge if the relaxation parameter is not carefully chosen [26].

For the boundary conditions we have used:

$$
\begin{aligned}
P_{\text {in }}(\mathbf{x}, t) & = \begin{cases}10^{3}(1-\cos (2 \pi t / 0.005)), & \mathbf{x} \in \Sigma_{1}, 0 \leq t \leq 0.005 \\
0, & \mathbf{x} \in \Sigma_{1}, 0.005 \leq t \leq T\end{cases} \\
\mathbf{g}(\mathbf{x}, t) & =0, \quad \mathbf{x} \in \Sigma_{2}, 0 \leq t \leq T
\end{aligned}
$$

and for the initial conditions we have taken: $u^{0}=0, \dot{u}^{0}=0, \ddot{u}^{0}=0, \mathbf{v}^{0}=0$.

The maximal pressure imposed at the inflow is $P_{i n}(\cdot, 0.0025)=2000 \frac{\text { dynes }}{\mathrm{cm}^{2}}$ and the time duration of the over-pressure at the inflow is $0.005 \mathrm{~s}$.

We have performed the simulation for $N=500$ time steps with a time step $\Delta t=0.0005 \mathrm{~s}$ which gives a time duration $T=N \Delta t=0.25 \mathrm{~s}$.

The numerical tests have been produced using freefem $++v 1.34$ (see [18]).

Only the first 5 modes have been considered for the structure. In order to compute the normal mode shapes, the system (1.6)-(1.9) has been solved using the software Maple. For the Newmark algorithm we have used $\delta=0.6$ and $\theta=0.5$.

For the approximation of the fluid velocity and pressure we have employed the triangular finite elements $\mathbb{P}_{1}+$ bubble and $\mathbb{P}_{1}$ respectively. We have used three reference meshes of parameters presented in Table 1.

The gradient of the cost function is approached by the Finite Differences Method with the grid spacing $\Delta \alpha_{k}=0.001$. 

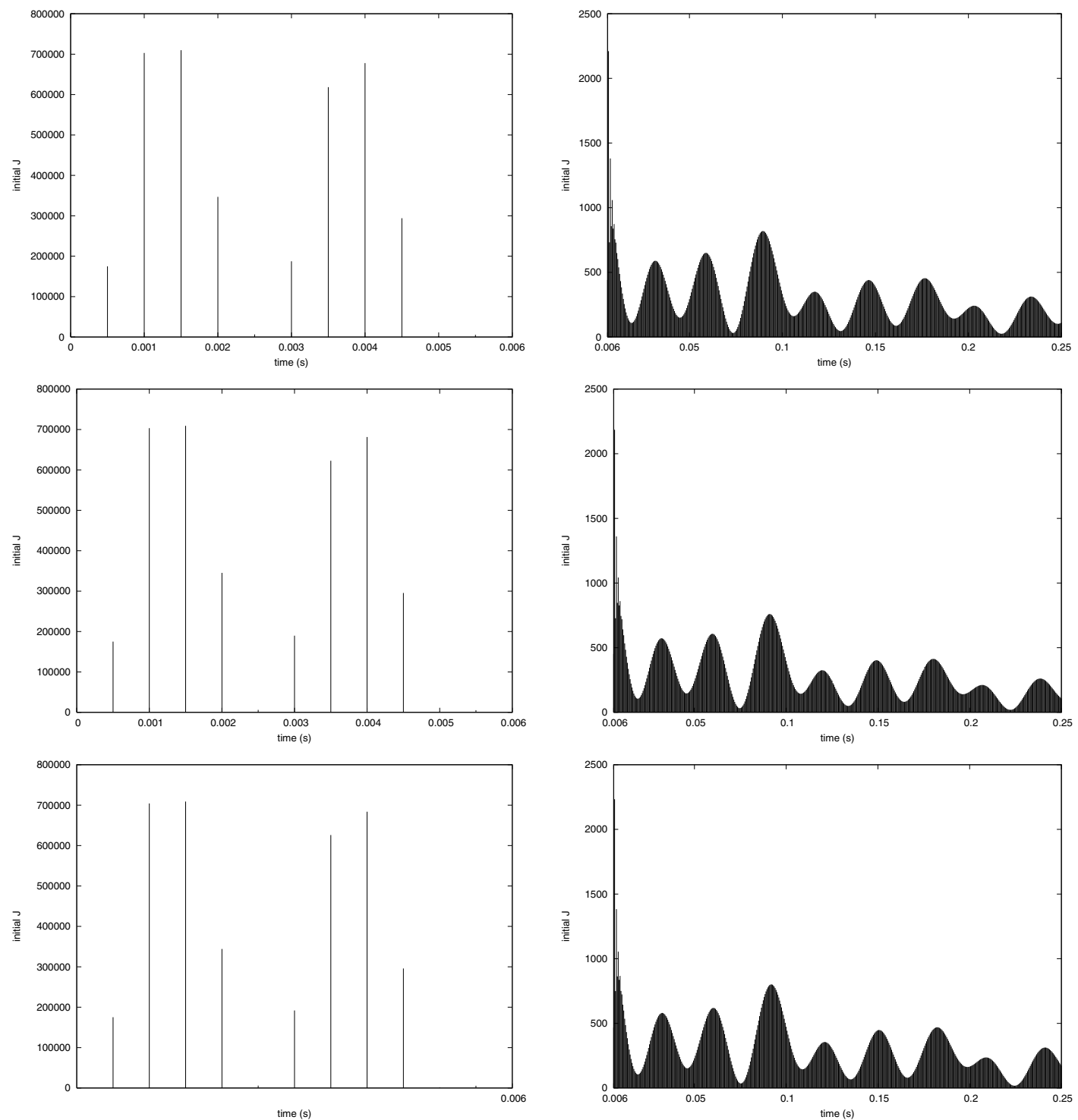

FIGURE 2. Starting values of the cost function during the pressure impulse (at the left) and after (at the right) for the mesh sizes $h_{1}$ (top), $h_{2}$ (middle), $h_{3}$ (bottom).

Starting point for the minimization algorithm. In our computations, the stress at the previous time step $\boldsymbol{\alpha}^{n}$ is used as starting point in the iterative method at the current time step. We remark in the left picture of Figure 2 that the starting values of the cost function are huge during the over-pressure imposed at the inflow. This means that the starting point is not closed to the solution, where the cost function reaches the zero value. Also, we observe that the starting values of the cost function are not very sensitive to the mesh size.

Stopping criteria and efficiency. At each time step, the optimization problem have been solved by the BFGS algorithm. 
We have employed the freefem ++ implementation of the BFGS algorithm which use the stopping criteria: $\|\nabla J\|<\epsilon$ or the number of iterations reaches a maximal value nbiter. We have performed the computations with $\epsilon=10^{-4}$ and nbiter $=8$. We set to 4 the maximal number of the iterations for the line search. For the Least Squares problems of zero residual, a more useful stopping criteria is $\|J\|<\epsilon$, but it is not implemented yet.

To sum up, at each time step the cost function is called at most

$$
\text { maximal number iterations BFGS } \times(m+\text { maximal number iterations line search }) .
$$

At each evaluation of the cost function we have to solve one structure sub-problem, to update the mesh and to solve one fluid sub-problem.

The final values of the cost function are less than $10^{-3}$ almost everywhere with very few exceptions (see Fig. 3).

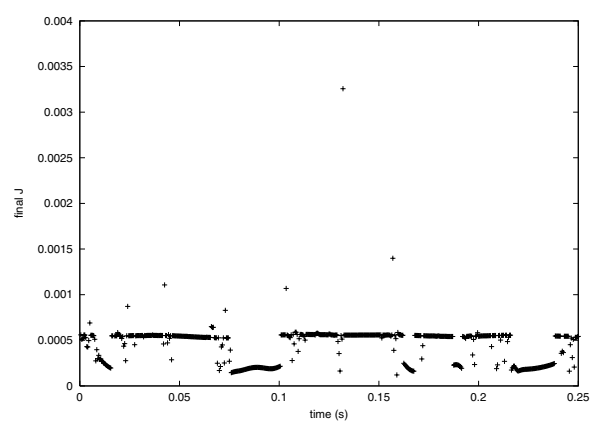

Figure 3. Final values of the cost function at each time step for the mesh size $h_{1}$.

From the formulas (3.46) and (3.47), it follows:

$$
\begin{aligned}
\|\mathrm{d} \dot{U}\|_{L^{2}(0, L)} & \leq \frac{(\Delta t) \delta}{\rho^{S} h^{S}} \sqrt{2 J^{n+1}(\boldsymbol{\alpha})} \leq \frac{0.0005 \cdot 0.6}{1.1 \cdot 0.1} \sqrt{2 \times 10^{-3}} \approx 1.21 \times 10^{-4} \\
\|\mathrm{~d} U\|_{L^{2}(0, L)} & \leq \frac{(\Delta t)^{2} \theta}{\rho^{S} h^{S}} \sqrt{2 J^{n+1}(\boldsymbol{\alpha})} \leq \frac{(0.0005)^{2} \cdot 0.5}{1.1 \cdot 0.1} \sqrt{2 \times 10^{-3}} \approx 5.08 \times 10^{-8} .
\end{aligned}
$$

In [26], at each time step, the coupled fluid-structure problem was solved by fixed point strategy with a relaxation parameter. At the start up of the simulation an important number of iterations (see [26], Fig. 4.14, p. 150) is necessary to satisfy the criteria

$$
\max \left(\frac{\|\mathrm{d} U\|_{L^{\infty}}}{\|U\|_{L^{\infty}}}, \frac{\|\mathrm{d} \dot{U}\|_{L^{\infty}}}{\|\dot{U}\|_{L^{\infty}}}\right) \leq 10^{-4} .
$$

The convergence of the fixed point algorithm can be accelerated using the Aitken's [14] or transpiration method [8].

Good convergence rate was obtained in [14] where the derivative of the operator was replaced by a much simpler operator. In order to achieve $\|\mathrm{d} U\| \leq 10^{-6}$, six iterations of quasi-Newton Method are required.

In [12], the block Newton algorithm is used where the Jacobian is evaluated exactly. The convergence is obtained in 2-3 iterations, only.

Behavior of the computed solution. The coupled fluid-structure algorithm is numerically stable for $\Delta t=0.0005 \mathrm{~s}$. 


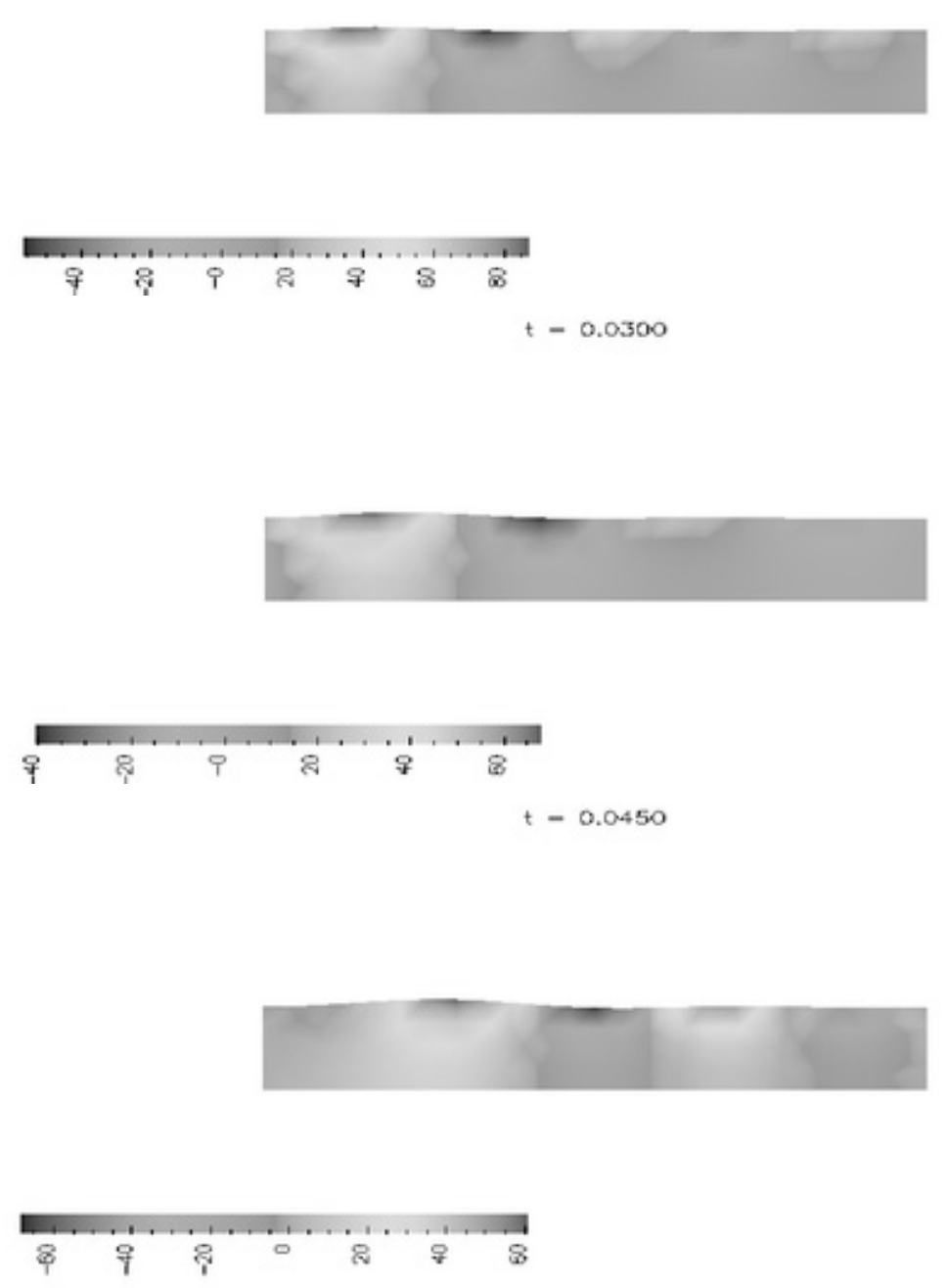

FiguRE 4. Fluid pressure $\left[\frac{\mathrm{dynes}}{\mathrm{cm}^{2}}\right]$.

The wave starts from the left side (see Figs. 4, 5) and it will be reflected at the right side. The animations including the displacement of the structure and the pressure of the fluid can be visualized on the web page of the author. The pulse speed is about $170 \mathrm{~cm} / \mathrm{s}$. In $[13,26]$, a 3D fluid-structure model is coupled with a $1 \mathrm{D}$ reduced model in order to reduce the reflexion due to the inappropriate boundary conditions for the structure and for the fluid on the right side.

\subsection{Case of a sine wave of the pressure input in a less compliant channel}

We will test now the sensitivity of the computed data by increasing the time step $\Delta t$. The BFGS Method will be successful from farther starting point. 


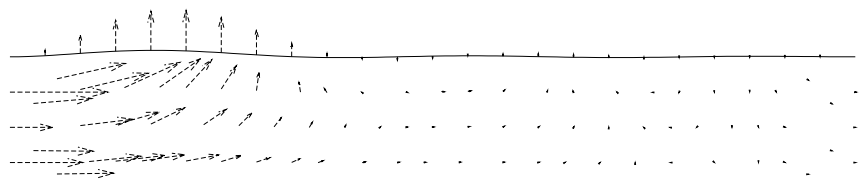

$\mathrm{t}=0.0300$

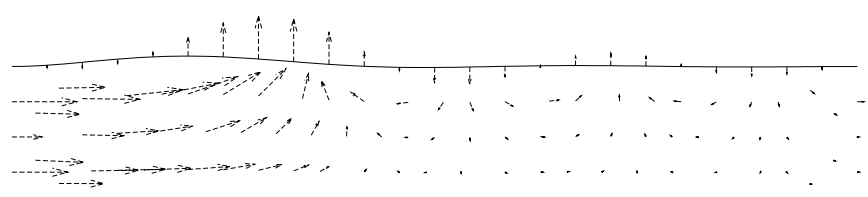

$\mathrm{t}=0.0450$

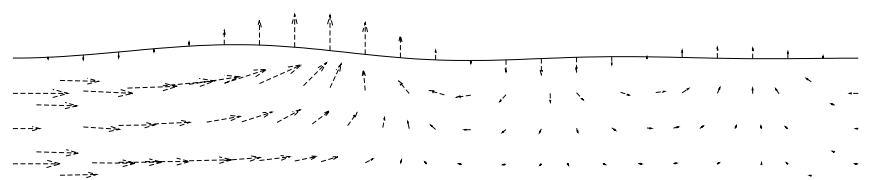

$t=0.0600$

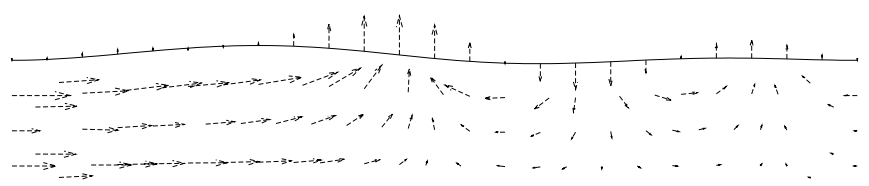

$t=0.0750$

Figure 5. Displacements $[\mathrm{cm}]$ of the top wall and fluid velocity $\left[\frac{\mathrm{cm}}{\mathrm{s}}\right]$. The arrows were scaled by a factor 0.1 .

The simulations were performed for $E=3 \times 10^{6} \frac{\mathrm{g}}{\mathrm{cm} \cdot \mathrm{s}^{2}}$ the Young modulus of the structure and for a five times longer over-pressure at the inflow:

$$
P_{i n}(\mathbf{x}, t)= \begin{cases}10^{3}(1-\cos (2 \pi t / 0.025)), & \mathbf{x} \in \Sigma_{1}, 0 \leq t \leq 0.025 \\ 0, & \mathbf{x} \in \Sigma_{1}, 0.025 \leq t \leq T\end{cases}
$$


TABLE 2. Parameters for the three numerical tests.

\begin{tabular}{|c|c|c|c|}
\hline$\Delta t$ & $h$ & $N$ & $T$ \\
\hline 0.0005 & 0.17 & 200 & 0.1 \\
\hline 0.0010 & 0.17 & 100 & 0.1 \\
\hline 0.0025 & 0.17 & 40 & 0.1 \\
\hline
\end{tabular}

The other parameters are the same as in the previous test.

For the three numerical tests, we have used the same reference mesh of 448 triangles and 267 vertices.

The time step $\Delta t$, the mesh size $h$, the number of time steps $N$ and $T=N \Delta t$ are reported in Table 2 .

The stress at the previous time step $\boldsymbol{\alpha}^{n}$ is used as starting point in the iterative method for solving the minimization problem $\min _{\boldsymbol{\alpha} \in \mathbb{R}^{m}} J^{n+1}(\boldsymbol{\alpha})$ at the current time step. The starting values of the cost function $J^{n+1}\left(\boldsymbol{\alpha}^{n}\right)$ are showed in the left column of Figure 6.

Remark that some values of the cost function in the starting points can reach $1.8 \times 10^{5}$ when $\Delta t=0.0005$ or $5 \times 10^{5}$ when $\Delta t=0.0010$ or $1.2 \times 10^{6}$ when $\Delta t=0.0025$. These great values mean that for some time instants, the solution at the previous time step is not closed to the solution at the current time step.

Also, we observe that values of the cost function in the starting points are very sensitive to $\Delta t$.

At each time step, the BFGS algorithm find efficiently an optimal value of the cost function less than 0.00055 (see the right column of Fig. 6).

We have performed less than 10 iterations of the BFGS algorithm and less than 5 iterations for the line search at each time step.

Even in the case of important displacements of the structure, the algorithm is successful (see Fig. 7).

\subsection{Modified Newton Method}

The Modified Newton Method (Newton Method with line search strategies) inherits the fast local convergence of the Newton Method and, in the same time, it is less sensitive to the starting point. We will see that, in some situations, this method can be a better choice than the BFGS Method.

Step 0. Choose a starting point $\boldsymbol{\alpha}^{0} \in \mathbb{R}^{m}$ and a positive scalar $\epsilon$. Set $k=0$.

Step 1. If $\left\|F\left(\boldsymbol{\alpha}^{k}\right)\right\|<\epsilon$ stop.

Step 2. Set $\mathbf{d}^{k}=-\left(\left(\nabla F\left(\boldsymbol{\alpha}^{k}\right)\right)^{T}\right)^{-1} F\left(\boldsymbol{\alpha}^{k}\right)$.

Step 3. Determine $\boldsymbol{\alpha}^{k+1}=\boldsymbol{\alpha}^{k}+\theta_{k} \mathbf{d}^{k}, \theta_{k}>0$ by means of an approximate minimization

$$
\left\|F\left(\boldsymbol{\alpha}^{k+1}\right)\right\| \approx \min _{\theta \geq 0}\left\|F\left(\boldsymbol{\alpha}^{k}+\theta \mathbf{d}^{k}\right)\right\| .
$$

Step 4. Update $k=k+1$ and go to the Step 1 .

In this paper, we make distinction between the Modified Newton Method described above and the Newton Method where at the Step 3, we always take $\theta_{k}=1$.

First, we will employ the Modified Newton Method for a nonlinear problem in $\mathbb{R}^{2}$, then for a fluid-structure interaction problem presented previously.

4.3.1. Validation tests

Let $F: \mathbb{R}^{2} \rightarrow \mathbb{R}^{2}$ be defined by

$$
F(\boldsymbol{\alpha})=F\left(\alpha_{1}, \alpha_{2}\right)=\left(\begin{array}{c}
\alpha_{1}^{2}+\alpha_{2}^{2}-2 \\
\exp \left(\alpha_{1}-1\right)+\alpha_{2}^{2}-2
\end{array}\right)
$$



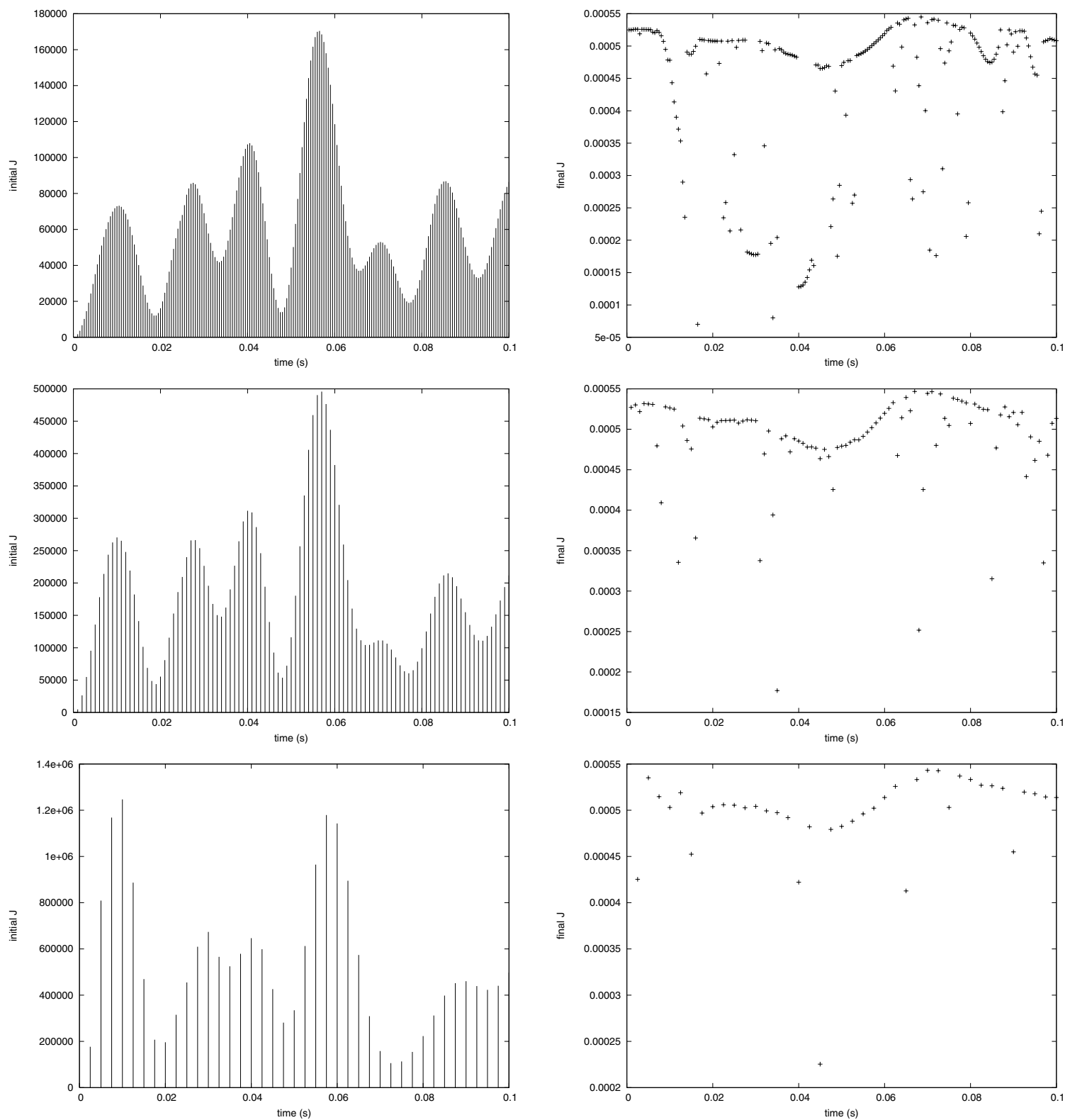

Figure 6. Starting (left) and final (right) values of the cost function for $\Delta t=0.0005$ (top), $\Delta t=0.0010$ (middle) and $\Delta t=0.0025$ (bottom).

which has the roots $(1,1)^{T}$ and $(1,-1)^{T}$. Numerical results obtained by Newton Method for solving $F(\boldsymbol{\alpha})=(0,0)^{T}$ are presented in [19]. The Jacobian of $F$ can be computed analytically by

$$
(\nabla F(\boldsymbol{\alpha}))^{T}=\left(\begin{array}{cc}
2 \alpha_{1} & 2 \alpha_{2} \\
\exp \left(\alpha_{1}-1\right) & 2 \alpha_{2}
\end{array}\right)
$$

Observe that the Jacobian is singular for $\alpha_{2}=0$. 


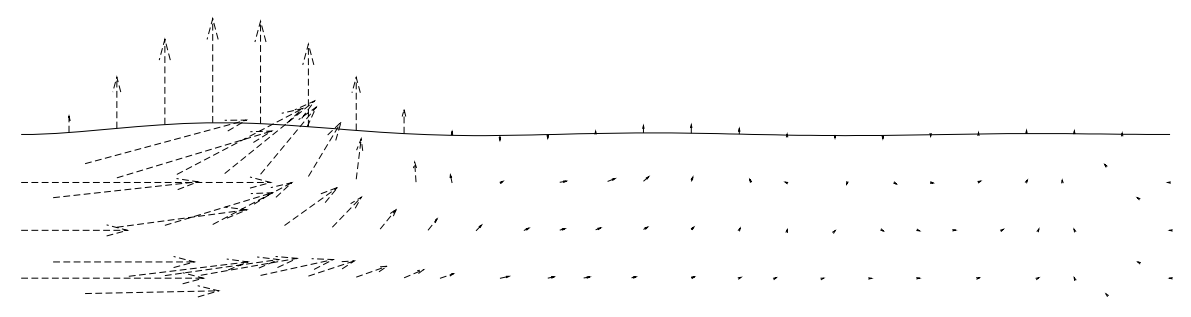

$t=0.0300$

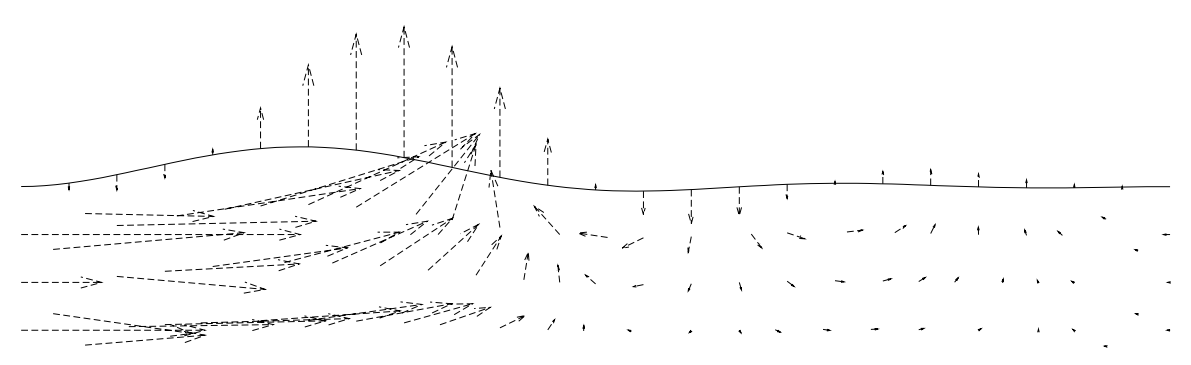

$t=0.0450$

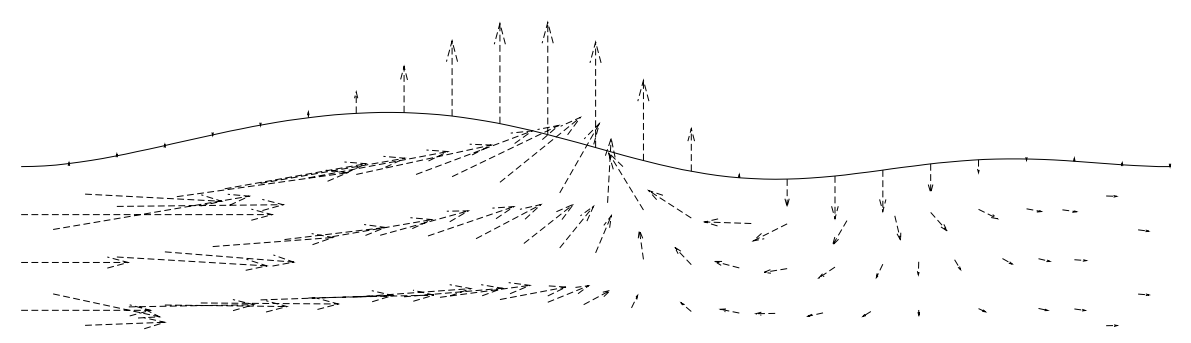

FiguRE 7 . Displacements $[\mathrm{cm}]$ of the top wall and fluid velocity $\left[\frac{\mathrm{cm}}{\mathrm{s}}\right]$. The arrows were scaled by a factor 0.05 .

We have investigated Newton, BFGS and Modified Newton Methods for two initial starting points: $\boldsymbol{\alpha}^{0}=(2,3)^{T}$ and $\boldsymbol{\alpha}^{0}=(3,5)^{T}$ using freefem $++[18]$. The line search strategy implemented in freefem ++ starts with the step $\theta=1$ and then, if it is not acceptable, reduces it using backtracking with cubic interpolation (see [6], Sect. 6.3.2, pp. 126-129).

We denote by $J(\boldsymbol{\alpha})=\frac{1}{2}\|F(\boldsymbol{\alpha})\|^{2}$, where $\|\cdot\|$ is the Euclidean norm of $\mathbb{R}^{2}$. The gradient can be computed analytically by $\nabla J(\boldsymbol{\alpha})=(\nabla F(\boldsymbol{\alpha})) F(\boldsymbol{\alpha})$. 
The first starting point: $\boldsymbol{\alpha}^{0}=(2,3)^{T}$

Newton Method. Only 4 iterations are needed to the Newton Method in order to satisfy the stopping criteria $\|F(\boldsymbol{\alpha})\|<\epsilon$, where $\epsilon=10^{-3}$. At the initial iteration we have $\boldsymbol{\alpha}^{0}=(2,3)^{T}, J\left(\boldsymbol{\alpha}^{0}\right)=107.723$. At the final iteration we have obtained: $\boldsymbol{\alpha}^{4}=(1,1.00011)^{T}, J\left(\boldsymbol{\alpha}^{4}\right)=5.01828 e-08$ and

$$
F\left(\boldsymbol{\alpha}^{4}\right)=(0.000224015,0.000224015)^{T} .
$$

BFGS Method. The stopping criteria $\|\nabla J(\boldsymbol{\alpha})\|<\epsilon$ for $\epsilon=10^{-3}$ is reached after 25 iterations. The mean number of evaluations of the cost function $J$ is 5.16 for the inaccurate line search. At the final iteration we have obtained: $\boldsymbol{\alpha}^{25}=(1.00015,-1.00036)^{T}, J\left(\boldsymbol{\alpha}^{25}\right)=9.05405 e-07$ and

$$
\nabla J\left(\boldsymbol{\alpha}^{25}\right)=(0.000299141,-0.000724457)^{T} .
$$

Modified Newton Method. We have performed the computations with the same line search strategy as in the BFGS Method. The stopping criteria $\|F(\boldsymbol{\alpha})\|<\epsilon$ holds after 12 iterations, where $\epsilon=10^{-3}$. The mean number of evaluations of the cost function $J$ is 4 for the inaccurate line search. At the final iteration we have obtained: $\boldsymbol{\alpha}^{12}=(0.999982,0.999824)^{T}, J\left(\boldsymbol{\alpha}^{12}\right)=1.42877 e-07$ and

$$
F\left(\boldsymbol{\alpha}^{12}\right)=(-0.000386687,-0.000369091)^{T} .
$$

Discussions. The Newton Method is the faster. The BFGS Method performs 25 iterations, while the Modified Newton Method only 12 to obtain a final cost function of about $10^{-7}$. The Newton and Modified Newton Methods approach the root $(1,1)^{T}$ and the BFGS Method finds the other root $(1,-1)^{T}$.

The second starting point: $\boldsymbol{\alpha}^{0}=(3,5)^{T}$

The Newton Method is divergent for this starting point. The history of the cost function is the following: $J\left(\boldsymbol{\alpha}^{0}\right)=974.747, J\left(\boldsymbol{\alpha}^{1}\right)=396.045, J\left(\boldsymbol{\alpha}^{2}\right)=1309.35$.

The Modified Newton Method will stagnate from the 9th to the 30th iteration near the point $\boldsymbol{\alpha}=(3.47282,0.000614253)^{T}$ which is not a solution because $J(\boldsymbol{\alpha})=99.1761$ and $F(\boldsymbol{\alpha})=(10.06,9.85592)^{T}$. This stagnation is a consequence of the fact that the Jacobian is singular for $\alpha_{2}=0$.

We have performed 30 iterations of the BFGS Method. The mean number of evaluations of the cost function $J$ for the inaccurate line search is 4.33. At the final iteration we have obtained: $\boldsymbol{\alpha}^{30}=(0.996168,-0.998812)^{T}$, $J\left(\boldsymbol{\alpha}^{30}\right)=6.9477 e-05$ and $\nabla J\left(\boldsymbol{\alpha}^{30}\right)=(0.00758799,0.002399)^{T}$.

Also, we have tested the BFGS Method with the starting point $\boldsymbol{\alpha}=(3.47282,0.000614253)^{T}$ which is a stagnation point for the Modified Newton Method. After the 21 iterations, the BFGS Method finds $\boldsymbol{\alpha}=(-0.47772,1.33111)$, where $J(\boldsymbol{\alpha})=3.62384 e-09$.

Discussions. If the starting points is not close to the solution, the Newton Method is divergent. Contrary to the Modified Newton Method, the BFGS Method gives satisfaction even in the neighborhood of the points where the Jacobian is singular.

\subsubsection{Solving fluid-structure interaction by Modified Newton Method}

In Section 4.2, we have presented numerical results for solving a fluid-structure interaction problem. At each time step, the BFGS Method was employed to solve inf $J(\boldsymbol{\alpha})=\frac{1}{2}\|F(\boldsymbol{\alpha})\|^{2}$.

The aim of this section is to compare the performances of the Modified Newton and BFGS Methods for solving a particular fluid-structure interaction problem. The stopping criteria for the Modified Newton Method $\|F(\boldsymbol{\alpha})\|<\epsilon$ is not equivalent to the one used by the BFGS Method $\|\nabla J\|=\|(\nabla F(\boldsymbol{\alpha})) F(\boldsymbol{\alpha})\|<\epsilon$, so we will proceed in the following manner: at each time step, we perform the same number of iterations of the both methods. We will compare the values of the cost function $J(\boldsymbol{\alpha})$ after 10 iterations. Then we will observe which method gives the smaller values. The same inexact line search strategy will be employed. 

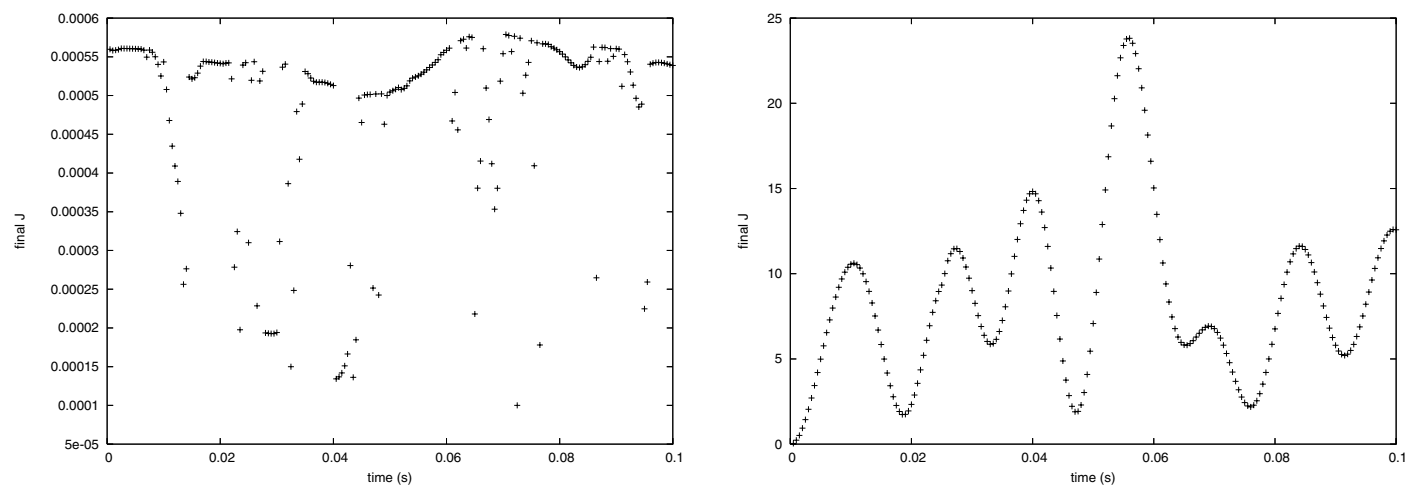

Figure 8. Final values of $J$ obtained by the BFGS (left) and Modified Newton Method (right) for $\Delta t=0.0005$.

\section{Moderate time step}

For the time step $\Delta t=0.0005$, the BFGS Methods finds final values of $J$ less than 0.0006 . The numerical results obtained by the Modified Newton Method are reported in the right plot of Figure 8. The BFGS Method wins at each time iteration.

Concerning the CPU time, in order to perform $N=200$ time iterations, the BFGS Method needs 2 hours, 30 minutes and 55 seconds while the Modified Newton Method needs 3 hours, 34 minutes and 38 seconds on a computer with two processors of $3.6 \mathrm{GHz}$ frequency.

The Jacobian of $F$ was computed by the same Finite Differences scheme as for $\nabla J$

$$
\frac{\partial F}{\partial \alpha_{k}}(\boldsymbol{\alpha}) \approx \frac{F\left(\boldsymbol{\alpha}+\Delta \alpha_{k} \mathbf{e}_{\mathbf{k}}\right)-F(\boldsymbol{\alpha})}{\Delta \alpha_{k}}
$$

where $\mathbf{e}_{\mathbf{k}}$ is the $k$-th vector of the canonical base of $\mathbb{R}^{m}$ and $\Delta \alpha_{k}>0$ is the grid spacing. The columns of the Jacobian are the vectors $\frac{\partial F}{\partial \alpha_{k}}$. In spite of the fact that the gradient of $J$ is a vector of dimension $m$ and the Jacobian of $F$ is a $m \times m$ matrix, the computation of the both needs the same number of evaluation of $F$, more precisely $m+1$. Contrary to the BFGS Method, at each iteration the Modified Newton Method requires the solution of a linear system in order to compute the direction $\mathbf{d}^{k}$.

Also, for the time step $\Delta t=10^{-4}$, the BFGS Method finds values of $J$ smaller than the Modified Newton Method.

\section{Small time step}

We have performed numerical tests for $N=100$ time iterations with the step $\Delta t=10^{-5}$. We can see in Figure 9, that the Modified Newton Method finds smaller values than the BFGS Method.

The CPU time is 76 minutes and 9 seconds for the BFGS Method and 107 minutes and 27 seconds for the Modified Newton Method.

After the time instant $t=0.0003$, the BFGS Method obtains final values of $J$ which have the first digits 0.0002955. The further digits change, but this is not visible on the left plot of Figure 9 .

Discussions. The numerical results presented in this section suggest to use the Modified Newton Method for small time steps, while the BFGS Method is preferable for moderate time steps.

\section{Conclusions}

In this paper, the continuity of the stresses at the interface was treated by the Least Squares Method. At each time step we have to solve an optimization problem which is less sensitive to the choice of the starting point and it permits us to use moderate time step. This is the main advantage of this approach. 

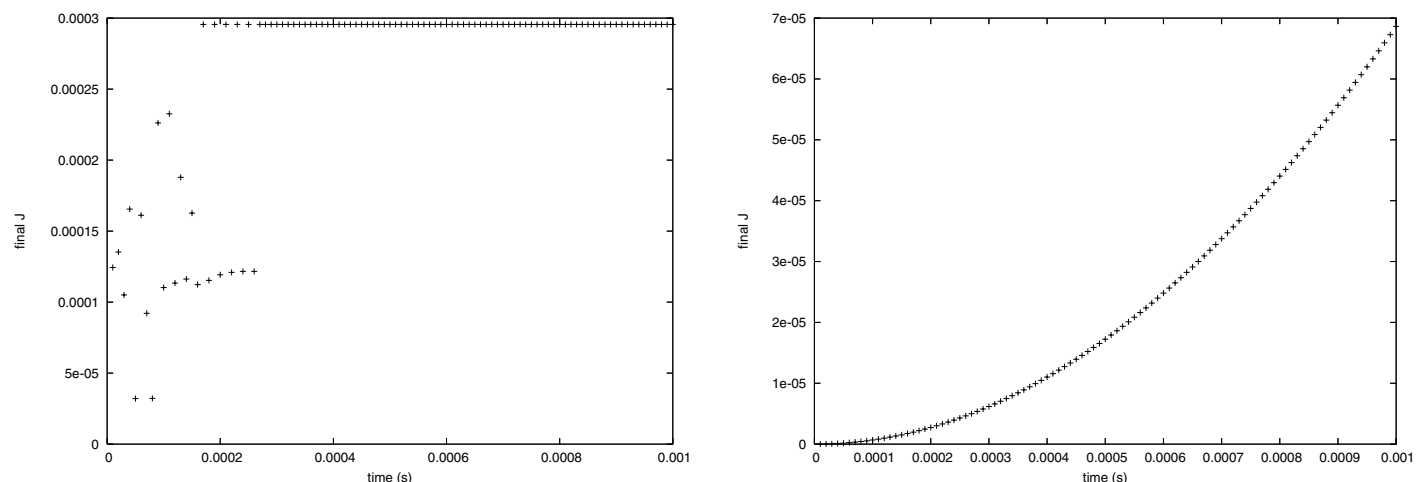

Figure 9. Final values of $J$ obtained by the BFGS (left) and Modified Newton Method (right) for $\Delta t=10^{-5}$.

For moderate time step, the solution at the previous time step is not close to the solution at the current time instant. Such phenomena is amplified during the phase when the flow rate increase or decrease rapidly or if we increase the time step. In order to solve the optimization problem, we have employed the BFGS Method which is successful from farther starting point. The gradient of the cost function was approached by the Finite Difference Method.

The coupled fluid-structure algorithm has good stability properties.

We conclude with a suggestion from [6]: use Newton like methods for their fast local convergence when ever it seems to be working well, otherwise use a slower method such BFGS but which is designed to converge to the local minimizer from almost any starting point.

Acknowledgements. The author is very grateful to the anonymous referees whose suggestions and comments have contributed to improve the content of this paper.

\section{REFERENCES}

[1] G. Bayada, M. Chambat, B. Cid and C. Vazquez, On the existence of solution for a non-homogeneous Stokes-rod coupled problem. Nonlinear Anal. Theory Methods Appl., 59 (2004) 1-19.

[2] H. Beirao da Veiga, On the existence of strong solution to a coupled fluid structure evolution problem. J. Math. Fluid Mech. 6 (2004) 21-52.

[3] P. Causin, J.F. Gerbeau, F. Nobile, Added-mass effect in the design of partitioned algorithms for fluid-structure problems, Comput. Methods Appl. Mech. Engrg. 194 (2005) 4506-4527.

[4] A. Chambolle, B. Desjardins, M.J. Esteban, C. Grandmont, Existence of weak solutions for an unsteady fluid-plate interaction problem. J. Math. Fluid Mech. 7 (2005) 368-404.

[5] R. Dautray and J.L. Lions, Analyse mathématique et calcul numérique pour les sciences et les techniques. Vol. 7, 9, Masson (1988).

[6] J.E. Dennis, Jr., and R.B. Schnabel, Numerical methods for unconstrained optimization and nonlinear equations. Classics in Applied Mathematics, 16, Society for Industrial and Applied Mathematics, Philadelphia, PA (1996).

[7] S. Deparis, Numerical Analysis of Axisymmetric Flows and Methods for Fluid-Structure Interaction Arising in Blood Flow Simulation, Ph.D. thesis, École Polytechnique Fédérale de Lausanne, Switzerland (2004).

[8] S. Deparis, M.A. Fernandez and L. Formaggia, Acceleration of a fixed point algorithm for fluid-structure interaction using transpiration conditions. ESAIM: M2AN 37 (2003) 601-616.

[9] B. Desjardins, M. Esteban, C. Grandmont and P. Le Tallec, Weak solutions for a fluid-elastic structure interaction model. Rev. Mat. Complut. 14 (2001) 523-538.

[10] G. Duvaut and J.-L. Lions, Les inéquations en mécanique et en physique. Dunod, Paris (1972).

[11] C. Farhat and M. Lesoinne, Two efficient staggered algorithms for the serial and parallel solution of three-dimensional nonlinear transient aeroelastic problems, Comput. Methods Appl. Mech. Engrg. 182 (2000) 499-515. 
[12] M.A. Fernandez and M. Moubachir, A Newton method using exact jacobians for solving fluid-structure coupling. Comput. Struct. 83 (2005) 127-142.

[13] L. Formaggia, J.F. Gerbeau, F. Nobile and A. Quarteroni, On the coupling of 3D and 1D Navier-Stokes equations for flow problems in compliant vessels. Comput. Methods Appl. Mech. Engrg. 191 (2001), 561-582.

[14] J.F. Gerbeau and M. Vidrascu, A quasi-Newton algorithm on a reduced model for fluid - structure interaction problems in blood flows. ESAIM: M2AN 37 (2003) 663-680.

[15] C. Grandmont, Existence for a three-dimensional steady state fluid-structure interaction problem. J. Math. Fluid Mech. 4 (2002) $76-94$.

[16] C. Grandmont and Y. Maday, Existence for an unsteady fluid-structure interaction problem. ESAIM: M2AN 34 (2000) 609-636.

[17] J.-L. Guermond and L. Quartapelle, On the approximation of the unsteady Navier-Stokes equations by finite element projection methods. Numer. Math. 80 (1998) 207-238.

[18] F. Hecht and O. Pironneau, A finite element software for PDE: freefem++, http://www.freefem.org.

[19] C.T. Kelley, Solving nonlinear equations with Newton's method. Fundamentals of Algorithms. Society for Industrial and Applied Mathematics (SIAM), Philadelphia, PA (2003).

[20] H.P. Langtangen, Computational Partial Differential Equations: numerical methods and Diffpack programming. Springer, Berlin (1999).

[21] P. Le Tallec, Introduction à la dynamique des structures, Cours École Polytechnique, Ellipses (2000).

[22] P. Le Tallec and J. Mouro, Fluid-structure interaction with large structural displacements. Comput. Methods Appl. Mech. Engrg. 190 (2001) 3039-3067.

[23] Y. Maday, B. Maury and P. Metier, Interaction de fluides potentiels avec une membrane élastique, in ESAIM Proc., Soc. Math. Appl. Indust., Paris 10 (1999) 23-33.

[24] C. Murea, The BFGS algorithm for a nonlinear least squares problem arising from blood flow in arteries. Comput. Math. Appl. 49 (2005) 171-186.

[25] C. Murea and C. Vazquez, Sensitivity and approximation of the coupled fluid-structure equations by virtual control method. Appl. Math. Optim. 52 (2005) 357-371.

[26] F. Nobile, Numerical approximation of fluid-structure interaction problems with application to haemodynamics. Ph.D. thesis, EPFL, Lausanne (2001).

[27] O. Pironneau, Conditions aux limites sur la pression pour les équations de Stokes et Navier-Stokes. C. R. Acad. Sc. Paris, 303 (1986) 403-406.

[28] A. Quarteroni and L. Formaggia, Mathematical Modelling and Numerical Simulation of the Cardiovascular System. Chapter in Modelling of Living Systems, N. Ayache Ed., Handbook of Numerical Analysis Series, Vol. XII, P.G. Ciarlet Ed., Elsevier, Amsterdam (2004).

[29] A. Quarteroni, M. Tuveri and A. Veneziani, Computational vascular fluid dynamics: problems, models and methods. Comput. Visual. Sci. 2 (2000) 163-197.

[30] J. Steindorf and H.G. Matthies, Partioned but strongly coupled iteration schemes for nonlinear fluid-structure interaction. Comput. Struct. 80 (2002) 1991-1999. 